\title{
SINGULAR INTEGRAL OPERATORS OVER A HILBERT SPACE
}

\author{
BY \\ MICHAEL J. FISHER(')
}

Introduction. Let $H$ denote a real separable Hilbert space and let $1<p<\infty$. Let $y \rightarrow T_{y}$ denote the regular representation of the additive group of $H$ acting on $L^{p}$ ( $H$, normal distribution). Thus if $f$ is a bounded tame function on $H$, then $\left(T_{y} f\right)(x)=f(x-y) D(x, y)$ where $D(x, y)=\exp \left[(x, y) / p-\|y\|^{2} / 2 p\right]$ is the $p$ th root of the Jacobian of translation by $y$. The $T_{y}$ are isometries on $L^{p}(H)$. If $\nu$ is a complex Borel measure on $H$ of bounded variation, let $\nu_{p}=\int_{H} T_{y} d \nu(y)$. It is the object of this paper to give sufficient conditions on a complex Borel measure $\mu$ of bounded variation on $H$ such that if $Z^{\delta \rho}(E)=\int_{\delta}^{o} \mu(E / t) d t / t$ for Borel sets $E$ and $0<\delta<\rho<\infty$, then the strong limit of the $Z_{p}^{\delta \rho}$ exists as a bounded operator on $L^{p}(H)$ as $\delta \rightarrow 0$ and $\rho \rightarrow \infty$.

A theorem of this type extends the Calderon-Zygmund theory of singular integral operators on $E_{n}$ to infinite dimensions. For if $k(y)\|y\|^{-n}$ is a CalderonZygmund kernel with $\nu(E)=\int_{E} k(y)\|y\|^{-n} d y$, then for $t>0, \nu(t E)=\nu(E)$ for bounded Borel sets $E$ which are disjoint from a neighborhood of the origin; and if $g(y)$ is an integrable radial function with support in a bounded annulus disjoint from a neighborhood of the origin, then $\int_{E_{n}} g(y) k(y)\|y\|^{-n} d y=0$. When $\mu$ satisfies a smoothness condition and $\mu(H)=0$, the set function $\nu(E)=\int_{0}^{\infty} \mu(E / t) d t / t$ has these properties.

If $\mu$ is an odd measure on $H$, the $Z_{p}^{\delta \rho}$ converge strongly (Theorem 7). If $\mu$ is an even measure of the form $d \mu(y)=a(y) d n \circ B^{-1}(y)$, where $B$ is a Hilbert-Schmidt operator, where $\mu(H)=0$, and where $a(y)$ satisfies certain smoothness conditions, the operators $Z_{p}^{\delta \rho}$ converge strongly (Theorem 8 and Corollaries 8.1, 8.2, and 8.3). When $p=2$, if $d \mu(y)=a(y) d n \circ B^{-1}(y)$ with $\mu(H)=0$ and $a(y) \in L^{r}\left(H, n \circ B^{-1}\right)$ for some $r>1$, the $Z_{2}^{\delta \rho}$ converge strongly (Theorem 5).

In $\S 5$ we shall make explicit the relationship between the singular integral operators of $\$ \S 3$ and 4 and the operators of Calderon and Zygmund (Theorem 9).

We apply our results to the study of the regularity properties of solutions of certain second order elliptic partial differential equations on the Hilbert space $H$.

In $\$ 7$ we give a brief sketch of certain generalizations of our main results to nonseparable Hilbert space.

The results in this paper are taken from the author's doctoral dissertation at

Presented in part to the Society, September 1, 1966 under the title $A n L^{2}$ theory for singular integrals on Hilbert space; received by the editors February 15, 1967.

(1) This research was supported in part by grants from the National Science Foundation. 
Cornell University, 1966. The author wishes to express his most hearty thanks to his thesis advisor, Professor Leonard Gross, for his interest, advice, and encouragement during the preparation of the dissertation.

1. The normal distribution on Hilbert space. The following definition is due to I. E. Segal $[9$, p. 116].

Definition 1.1. A weak distribution on a real Hilbert space $H$ is an equivalence class of linear maps, $F$, from $H^{*}$ to measurable functions on a probability space $(S, \Sigma, \mu)$ (depending on $F$ ). Two such maps, $F_{1}$ and $F_{2}$, are equivalent if for each finite set of vectors $y_{1}, \ldots, y_{n}$ in $H^{*}$, the joint distributions of $F_{j}\left(y_{1}\right), \ldots, F_{j}\left(y_{n}\right)$ in $n$-space are equal for $j=1,2$. A weak distribution is continuous if one of its representatives is a continuous linear map (the range spaces have the topology of convergence in measure).

In [9, pp. 116-118] Segal formulated the theory of integration with respect to weak distributions. He has shown that meaning can be given to the concepts of measurability, $p$-power integrability, and convergence in measure which is relevant for the weak distribution and not dependent on the representative chosen. If $n$ and $n^{\prime}$ are representatives of a weak distribution on $H$, then $n$ and $n^{\prime}$ are maps from $H^{*}$ to measurable functions on probability spaces $(S, \Sigma, \mu)$ and $\left(S^{\prime}, \Sigma^{\prime}, \mu^{\prime}\right)$. Let $\Sigma_{0}$ denote the smallest $\sigma$-ring in $\Sigma$ with respect to which all of the functions $n(y)(\cdot)$ are measurable for $y \in H^{*}$ and let $\Sigma_{0}^{\prime}$ be the similarly defined sub- $\sigma$-ring of $\Sigma^{\prime}$. Let $M$ be the algebra of bounded complex-valued $\Sigma_{0}$-measurable functions on $S$ modulo the ideal of null functions. We now identify elements of $M$ with functions on $S$ in the usual way and set $E(f)=\int_{S} f d \mu$ for $f \in M$. Let $M^{\prime}$ be the similarly defined algebra on $\left(S^{\prime}, \Sigma_{0}^{\prime}, \mu^{\prime}\right)$ and let $E^{\prime}$ be the similarly defined expectation functional on $M^{\prime}$. Segal $[9$, p. 117] has shown that there is a unique expectation preserving isomorphism $\phi$ from $M$ to $M^{\prime}$ (i.e. $E(f)=E^{\prime}(\phi(f))$ ) whose extension to the algebra of all $\Sigma_{0}$-measurable functions on $S$ maps $n(y)$ into $n^{\prime}(y)$. Thus the weak distribution determines an essentially unique (i.e. up to expectation preserving isomorphism) algebra $M$-the bounded algebra of $H$ with respect to the weak distribution-and an expectation functional, $E$, on $M$.

In this paper we will be primarily concerned with the normal distribution on $H$; that is, the unique distribution, $n$, on $H$ with the following properties:

(1) For any vector $x \in H, n(x)$ is normally distributed with mean zero and variance $c\|x\|^{2}$ for $c>0$.

(2) $n$ maps orthogonal vectors into independent random variables. The parameter $c$ will be taken to be equal to 1 in this paper since the generalization of our results to the case $c \neq 1$ is trivial. We may define $L^{p}(H)$ with respect to the normal distribution as follows: Denote by $N$ the bounded algebra of $H$ with respect to the normal distribution on $H$ and let $E$ be the expectation functional on $N$. For $1 \leqq p<\infty, L^{p}(H)$ is the completion of $N$ in the norm $\|f\|_{p}=E\left(|f|^{p}\right)^{1 / p}$ and we set $N=L^{\infty}(H) . L^{p}(H)$ may be identified with any of the spaces $L^{p}\left(S, \Sigma_{0}, \mu\right)$ when the map 
$F$ from $H^{*}$ to measurable functions on $\left(S, \Sigma_{0}, \mu\right)$ is a representative of the normal distribution and $\Sigma_{0}$ contains no smaller $\sigma$-ring with respect to which all the $F(y)(\cdot)$ are measurable. We refer to the random variables on the essentially unique probability space $\left(S, \Sigma_{0}, \mu\right)$ as the measurable functions on $H$ with respect to the normal distribution.

In what follows the weak distribution under consideration is the normal distribution on $H$. A complex valued function $f(x)$ on $H$ is a tame function if there exists a finite dimensional projection $P$ on $H$ and a Baire function $g$ on the range of $P$ such that $f(x)=g(P x)$. A tame function, $f$, determines a measurable tame function, $\tilde{f}$, on $H$ in the following way: If $F$ is a representative of the normal distribution on $H$ and $f(x)=g(P x)=g\left(\left\langle x, e_{1}\right\rangle, \ldots,\left\langle x, e_{k}\right\rangle\right)$ then $\tilde{f}(\omega)=g\left(F\left(e_{1}\right)(\omega)\right.$, $\left.\ldots, F\left(e_{k}\right)(\omega)\right)$. We will often not distinguish between $f$ and $f$ when $f$ is a tame function on $H$.

It is not the case that all measurable functions on $H$ can be realized as functions on $H$ and not all bounded uniformly continuous functions on $H$ determine measurable functions on $H$. Let us denote by $\mathscr{F}$ the directed set of finite dimensional projections on $H$ directed by inclusion of the ranges; let $f$ be a complex valued function on $H$ such that $f \circ P$ is a tame function for each $P \in \mathscr{F}$, and consider the net of measurable tame functions $\left\{(f \circ P)^{\sim} \mid P \in \mathscr{F}\right\}$. If this net converges in measure as $P$ tends strongly to the identity, $I$, through $\mathscr{F}$, then the limit, $f$, is a measurable function on $H$ whose integration properties are determined by the function $f$ and the normal distribution, $n$. Under certain continuity conditions on the function $f$, the net $\left\{(f \circ P)^{\sim} \mid P \in \mathscr{F}\right\}$ of measurable tame functions converges.

Definition 1.2 (Gross). $H_{2}$ is the weakest topology on $H$ for which all HilbertSchmidt operators are continuous from $\mathrm{H}_{2}$ to the strong topology on $H$.

Gross [6, Theorem 1, p. 406] has shown that if a complex valued function $f$ on $H$ is uniformly continuous near zero in the topology $H_{2}$, then the net $\left\{(f \circ P)^{\sim} \mid P \in \mathscr{F}\right\}$ of measurable tame functions converges in measure as $P$ tends strongly to the identity through $\mathscr{F}$ to a measurable function $\tilde{f}$. Furthermore, if $f$ is not identically zero on $H, \tilde{f}$ is not a null function.

There is a more general continuity condition on functions $f$ on $H$ which guarantees that the net $\left\{(f \circ P)^{\sim} \mid P \in \mathscr{F}\right\}$ converges as $P$ tends strongly to the identity through $F$. This condition will be useful in studying the Fourier transforms of singular integral operators on $L^{2}(H)$.

Definition 1.3. A complex valued function, $f$, on $\mathrm{H}$ is uniformly $\mathrm{H}_{2}$-continuous on $H_{2}$-bounded sets of $H-\{0\}$ if there exist sequences of Hilbert-Schmidt operators $\left\{B_{k}\right\}$ and $\left\{C_{j}\right\}$ such that the measurable functions $\left\|B_{k}(\cdot)\right\|^{\sim}$ (which exist by Lemma 1.2, p. 406 of [6]) tend to infinity in measure as $k$ tends to infinity and $\left\|C_{j}\right\|_{2}=\operatorname{tr}\left(C_{j}^{*} C_{j}\right)^{1 / 2}$ tends to zero as $j$ tends to infinity such that $f(x)$ is uniformly $H_{2}$-continuous on the sets $E_{j k}=\left\{x \mid\left\|C_{j} x\right\|<1\right\} \cap\left\{x \mid\left\|B_{k} x\right\|>1\right\}$ for $1 \leqq j, k<\infty$. 
THEOREM 1. Let $f$ be a complex valued function on $\mathrm{H}$ which is uniformly $\mathrm{H}_{2^{-}}$ continuous on $\mathrm{H}_{2}$-bounded sets of $H-\{0\}$. Then the net $\left\{(f \circ P)^{\sim} \mid P \in \mathscr{F}\right\}$ of measurable tame functions converges in measure as $P$ tends strongly to the identity through $\mathscr{F}$ to a measurable function $\tilde{f}$. If $f$ is bounded on $H$, and if there exists an operator $B_{K}$ (from the sequence $\left\{B_{k}\right\}$ of Definition 1.3) such that there is an $x_{0}$ in $H$ with $f\left(x_{0}\right) \neq 0$ and $\left\|B_{K} x_{0}\right\|>1+\varepsilon$ for some $\varepsilon>0$, then $\tilde{f}$ is not a null function.

Proof. For $\delta, \varepsilon>0$, we shall determine $P_{0} \in \mathscr{F}$ such that if $P, Q \geqq P_{0}$, and $P$, $Q \in \mathscr{F}$ then Meas $\left(\left|f(P x)^{\sim}-f(Q x)^{\sim}\right| \geqq \varepsilon\right)<\delta$. Let $\left\{B_{k}\right\}$ and $\left\{C_{j}\right\}$ be the sequences of Hilbert-Schmidt operators in Definition 1.3. Then for $d>0$ and $K>0$ there is an integer $N_{0}$ such that Meas $\left(\left\|B_{n}(\cdot)\right\| \sim \leqq K\right)<d$ for $n \geqq N_{0}$ since $\left\|B_{n}(\cdot)\right\| \sim$ tends to infinity in measure as $n$ tends to infinity. Let $N \geqq N_{0}$ be a fixed integer such that Meas $\left(\left\|B_{N}(\cdot)\right\| \sim \leqq 3 / 2\right)<\delta / 10$. Since $\left\|C_{j}\right\|_{2} \rightarrow 0$ as $j \rightarrow \infty,\left\|C_{j}(\cdot)\right\|^{\sim} \rightarrow 0$ in $L^{2}(H)$ as $j \rightarrow \infty$ by Lemma 1.2 of [6]. Hence $\left\|C_{j}(\cdot)\right\|^{\sim} \rightarrow 0$ in measure as $j \rightarrow \infty$. Let $J_{0}$ be sufficiently large that for $j \geqq J_{0}$, Meas $\left(\left\|C_{j}(\cdot)\right\| \sim \geqq 1 / 2\right)<\delta / 10$. Fix $J \geqq J_{0}$. By Lemma 1.2 of [6], the nets of tame functions $\left\{\left\|B_{N} P(\cdot)\right\|^{\sim} \mid P \in \mathscr{F}\right\}$ and $\left\{\left\|C_{J} P(\cdot)\right\|^{\sim} \mid P \in \mathscr{F}\right\}$ converge in measure to $\left\|B_{N}(\cdot)\right\|^{\sim}$ and $\left\|C_{J}(\cdot)\right\| \sim$, respectively, as $P$ tends strongly to the identity through $\mathscr{F}$. Therefore, there is $R \in \mathscr{F}$ such that for $P \in \mathscr{F}$ and $P \geqq R$, Meas $\left(\left|\left\|C_{J}(\cdot)\right\|^{\sim}-\left\|C_{J} P(\cdot)\right\| \sim\right| \geqq 1 / 2\right)<\delta / 10$ and Meas $\left(\left|\left\|B_{N}(\cdot)\right\|^{\sim}-\left\|B_{N} P(\cdot)\right\| \sim\right|\right.$ $\geqq 1 / 2)<\delta / 10$. Then Meas $\left(\left\|C_{J} P(\cdot)\right\| \sim \geqq 1\right)<\delta / 5$ and Meas $\left(\left\|B_{N} P(\cdot)\right\| \sim 1\right)<\delta / 5$ for $P \geqq R$ in $\mathscr{F}$. Since $f(x)$ is uniformly $H_{2}$-continuous on $E_{J N}=\left\{x \mid\left\|B_{N} x\right\|>1\right\}$ $\cap\left\{x \mid\left\|C_{J} x\right\|<1\right\}$, there is a Hilbert-Schmidt operator $A$ such that if $\left\|B_{N} x\right\|>1$, $\left\|B_{N} y\right\|>1,\left\|C_{J} x\right\|<1,\left\|C_{J} y\right\|<1$, and $\|A(x-y)\|<1$, then $|f(x)-f(y)|<\varepsilon$. There exists $S \in \mathscr{F}$ such that if $P, Q \geqq S$ for $P$ and $Q$ in $\mathscr{F}$, then $\|A(P-Q)\|_{2}<(\delta / 5)^{1 / 2}$, so that Meas $(\|A(P-Q)(\cdot)\| \sim \geqq 1)<\delta / 5$. Let $P_{0}=R \vee S$ (taking the sup in $\mathscr{F}$ ). Since $|f(P x)-f(Q x)|<\varepsilon$ when $\left\|B_{N} P x\right\|>1,\left\|B_{N} Q x\right\|>1,\left\|C_{J} P x\right\|<1,\left\|C_{J} Q x\right\|<1$, and $\|A(P-Q) x\|<1$, we have that

$$
\begin{aligned}
& \text { Meas }\left(\left|f(P x)^{\sim}-f(Q x)^{\sim}\right| \geqq \varepsilon\right) \\
& \leqq \text { Meas }\left(\left\|B_{N} P x\right\|^{\sim} \leqq 1\right)+\text { Meas }\left(\left\|B_{N} Q x\right\|^{\sim} \leqq 1\right)+\operatorname{Meas}\left(\left\|C_{J} P x\right\|^{\sim} \geqq 1\right) \\
& + \text { Meas }\left(\left\|C_{J} Q x\right\| \sim \geqq 1\right)+\text { Meas }(\|A(P-Q) x\| \sim \geqq 1) \\
& \leqq 5 \delta / 5=\delta \text {. }
\end{aligned}
$$

Since the space of measurable functions with the topology of convergence in measure is a complete metric space, it follows that there exists a measurable function $\tilde{f}$ on $H$ such that the net $\left\{(f \circ P)^{\sim} \mid P \in \mathscr{F}\right\}$ of measurable tame functions converges to $\tilde{f}$ in measure as $P$ tends strongly to the identity through $\mathscr{F}$.

To prove the second statement of the theorem, let $g(t)$ be a continuously differentiable function on the positive real line such that $g(t)=0$ for $t \leqq 1, g(t)=1$ for $t>1+\varepsilon$, and $0 \leqq g(t) \leqq 1$ on all of $t \geqq 0$. Then if $B_{K}$ is the operator in the statement of the theorem, consider $h(x)=g\left(\left\|B_{K} x\right\|\right) f(x)$. Then $h\left(x_{0}\right) \neq 0$ and $h(x)$ is uniformly continuous near zero in the topology $\mathrm{H}_{2}$. Thus by the first statement of the theorem, $h$ determines a measurable function $\tilde{h}=\operatorname{limit}$ in measure $(h \circ P)^{\sim}$ 
as $P$ tends strongly to the identity through $\mathscr{F}$. But $(h \circ P)^{\sim}(\cdot)=\tilde{g}\left(\left\|B_{N} P(\cdot)\right\|\right)(f \circ P)^{\sim}(\cdot)$ and thus $\tilde{h}(\cdot)=\tilde{g}\left(\left\|B_{N}(\cdot)\right\|\right) \tilde{f}(\cdot)$. By Corollary 1.1 of [6], $\tilde{h}$ is not a null function, thus $\tilde{f}$ is not a null function.

THEOREM 2. Let $f_{n}$ be a sequence of complex valued functions on a Hilbert space, $H$, which are uniformly $H_{2}$-continuous on all of $H$. Let $\left\{B_{k}\right\}$ and $\left\{C_{j}\right\}$ be sequences of Hilbert-Schmidt operators such that $\left\|B_{k}(\cdot)\right\| \sim$ tends to infinity in measure as $k$ tends to infinity and $\left\|C_{j}\right\|_{2}$ tends to zero as $j$ tends to infinity. Assume that the $f_{n}$ converge uniformly to $f$ on $E_{j k}=\left\{x \mid\left\|C_{j} x\right\|<1\right\} \cap\left\{x \mid\left\|B_{k} x\right\|>1\right\}$ for $1 \leqq j, k<\infty$. Then $f(x)$ is uniformly $\mathrm{H}_{2}$-continuous on the sets $E_{j k}$, and $f$ is uniformly $\mathrm{H}_{2}$-continuous on $\mathrm{H}_{2}$-bounded sets of $\mathrm{H}-\{0\}$. The corresponding measurable functions $\tilde{f}$ and $\tilde{f}_{n}$ (which exist by Theorem 1) satisfy $\tilde{f}=\operatorname{limit}_{n \rightarrow \infty}$ in measure $\tilde{f}_{n}$.

Proof. An $\varepsilon / 3$-argument shows that the limit $f(x)$ of the $f_{n}(x)$ is uniformly $\mathrm{H}_{2}$ continuous on the sets $E_{j k}$ and by Definition $1.3, f(x)$ is uniformly $H_{2}$-continuous on $H_{2}$-bounded sets of $H-\{0\}$. By Theorem $1, f$ determines a measurable function $\tilde{f}$ on $H$.

For $\delta, \varepsilon>0$, we shall show that there is an integer $N$ such that $n \geqq N$ implies Meas $\left(\left|\tilde{f}_{n}-\tilde{f}\right| \geqq \varepsilon\right)<\delta$. Since $\left\|B_{k}(\cdot)\right\|^{\sim} \rightarrow \infty$ in measure as $k \rightarrow \infty$, there is an integer $K_{0}$ such that if $k \geqq K_{0}$ then Meas $\left(\left\|B_{k}(\cdot)\right\| \sim 3 / 2\right)<\delta / 8$. Fix $k=K \geqq K_{0}$. By Lemma 1.2 of [6], there exists $R$ in $\mathscr{F}$ such that Meas $\left(\left|\left\|B_{K}(\cdot)\right\| \sim-\left\|B_{K} P(\cdot)\right\| \sim\right| \geqq 1 / 2\right)$ $<\delta / 8$ for $P$ in $\mathscr{F}$ and $P \geqq R$. Then Meas $\left(\left\|B_{K} P x\right\| \sim \leqq 1\right)<\delta / 4$. Since $\left\|C_{j}\right\|_{2} \rightarrow 0$ as $j \rightarrow \infty,\left\|C_{j}(\cdot)\right\|^{\sim} \rightarrow 0$ in measure by Lemma 1.2 of [6]. Choose $J_{0}$ sufficiently large that Meas $\left(\left\|C_{j}(\cdot)\right\| \sim \geqq 1 / 2\right)<\delta / 8$ when $j \geqq J_{0}$. Fix $j=J \geqq J_{0}$. By Lemma 1.2 of [6], there is $R_{1}$ in $\mathscr{F}$ such that if $P$ is in $\mathscr{F}$ and $P \geqq R_{1}$ then

$$
\text { Meas }\left(\left|\left\|C_{J}(\cdot)\right\| \sim-\left\|C_{J} P(\cdot)\right\| \sim\right| \geqq 1 / 2\right)<\delta / 8 .
$$

Then Meas $\left(\left\|C_{J} P x\right\| \sim \geqq 1\right)<\delta / 4$ for $P \geqq R_{1}$. Let $E_{J K}=\left\{x \mid\left\|C_{J} x\right\|<1\right\} \cap\left\{x \mid\left\|B_{K} x\right\|>1\right\}$. Choose $N$ sufficiently large that $\left|f_{n}(x)-f(x)\right|<\varepsilon / 3$ when $x \in E_{J K}$ and $n \geqq N$. Fix $n \geqq N$. There is a projection $S$ in $\mathscr{F}$ such that if $P \in \mathscr{F}$ and $P \geqq S$, then

$$
\text { Meas }\left(\left|\tilde{f}-(f \circ P)^{\sim}\right| \geqq \varepsilon / 3\right)<\delta / 4 \text { and } \text { Meas }\left(\left|\tilde{f}_{n}-\left(f_{n} \circ P\right)^{\sim}\right| \geqq \varepsilon / 3\right)<\delta / 4 \text {. }
$$

Now,

$$
\begin{aligned}
\operatorname{Meas}\left(\left|\tilde{f}-\tilde{f}_{n}\right| \geqq \varepsilon\right) \leqq & \operatorname{Meas}\left(\left|\tilde{f}_{n}-\left(f_{n} \circ P\right)^{\sim}\right| \geqq \varepsilon / 3\right) \\
& + \text { Meas }\left(\left|(f \circ P)^{\sim}-\left(f_{n} \circ P\right)^{\sim}\right| \geqq \varepsilon / 3\right) \\
& + \text { Meas }\left(\left|(f \circ P)^{\sim}-\tilde{f}\right| \geqq \varepsilon / 3\right) .
\end{aligned}
$$

Choose $P \geqq R_{1} \vee S \vee R$ (taking the sup in $\mathscr{F}$ ). The first and third terms on the right are each $<\delta / 4$. To estimate the second norm, note that $\left|f_{n}(P x)-f(P x)\right| \geqq \varepsilon / 3$ implies that $\left\|B_{K} P x\right\| \leqq 1$ or $\left\|C_{J} P x\right\| \geqq 1$. Thus

$$
\text { Meas } \begin{aligned}
\left(\left|(f \circ P)^{\sim}-\left(f_{n} \circ P\right)^{\sim}\right| \geqq \varepsilon / 3\right) \leqq & \operatorname{Meas}\left(\left\|B_{K} P x\right\|^{\sim} \leqq 1\right) \\
& + \text { Meas }\left(\left\|C_{J} P x\right\|^{\sim} \geqq 1\right)<2 \delta / 4 .
\end{aligned}
$$

Therefore, Meas $\left(\left|\tilde{f}_{n}-\tilde{f}\right| \geqq \varepsilon\right)<\delta$ for $n \geqq N$. 
EXAMPLes. 1. Any function $f$ on $H$ which is uniformly continuous near zero in the topology $\mathrm{H}_{2}$ is uniformly $\mathrm{H}_{2}$-continuous on $H_{2}$-bounded sets of $H-\{0\}$. For there exists a sequence $\left\{A_{n}\right\}$ of Hilbert-Schmidt operators on $H$ such that $\left\|A_{n}\right\|_{2}$ tends to zero as $n$ tends to infinity and $f$ is uniformly continuous on the sets $\left\{x \mid\left\|A_{n} x\right\|<1\right\}$ for $1 \leqq n<\infty$. Let $C_{j}=A_{j}$ and $B_{k}=k A_{1}$.

2. Let $A$ be a Hilbert-Schmidt operator on $H$ and let $p(y)$ be a polynomial on $H$. Define $f(x)=p\left(A x\|A x\|^{-1}\right)$ if $A x \neq 0$ and $f(x)=0$ if $A x=0$. Then successive applications of the triangle and Schwartz inequalities show that it suffices to show that for each $e \in H(A x, e)\|A x\|^{-1}$ is uniformly $H_{2}$-continuous on $H_{2}$-bounded sets of $H-\{0\}$ in order to prove that $f(x)$ has this property. Set $C_{j}=j^{-1} A$ and $B_{k}=k A$; the fact that $(A x, e)\|A x\|^{-1}$ has the desired continuity property is easily established. In addition, the set, $C h_{0}^{A}(H)$, of uniform limits of the above functions, $f(x)=$ $p\left(A x\|A x\|^{-1}\right)$ for $A x \neq 0$ and $f(x)=0$ for $A x=0$, consists of functions which are uniformly $\mathrm{H}_{2}$-continuous on $\mathrm{H}_{2}$-bounded sets of $H-\{0\}$.

When the operator $A$ is suitably chosen, the function space $C h_{0}^{A}(H)$ in Example 2 will contain the Fourier transforms of the singular integral operators on $L^{2}(H)$ which will be introduced in $\S 3$.

2. Harmonic analysis on a Hilbert space. Throughout this section and in the following sections of this paper, $H$ will denote a real separable Hilbert space which is generally infinite dimensional.

In this section we list the facts concerning harmonic analysis over the Hilbert space $H$ which will be most useful to us later. Denote by $M(H)$ the set of all complex valued Borel measures of bounded variation on $H$. The Fourier transform, $\hat{\mu}(y)$, of a measure $\mu$ in $M(H)$ is the function

$$
\hat{\mu}(y)=\int_{H} \exp [i(y, x)] d \mu(x) .
$$

Gross [7, Theorem 1, p. 7] has shown that if $\mu$ is a complex Borel measure of finite total variation on $H$, then its Fourier transform, $\hat{\mu}(y)$, is uniformly continuous on all of $H$ in the topology $H_{2}$. Thus, for any real constant $a$, by Theorem 1, $\hat{\mu}($ ay $)$ determines a measurable function $\tilde{\mu}(a \cdot)$ on $H$ and $\tilde{\mu}(a \cdot)=$ limit in measure $(\mu \circ P)^{\sim}(a \cdot)$ as $P$ tends strongly to the identity through the directed set $F$ of finite dimensional projections on $H$. With convolution as multiplication, $M(H)$ is a commutative Banach algebra with unit.

We shall consider operators on $L^{p}(H), 1 \leqq p<\infty$, which generalize the convolution operators

$$
U(f)(x)=\int_{E_{N}} f(x-y) d \mu(y),
$$

$\mu \in M\left(E_{N}\right)$, over finite dimensional Euclidean spaces. For $y \in H$ and $1 \leqq p<\infty$, consider the function $D(x, y)=\exp \left[(x, y) / p-\|y\|^{2} / 2 p\right]$. For each $y \in H, D(x, y)$ is a tame function based on the line through $y$. Segal [10, Theorem 3, pp. 22-23] has 
shown that the function $\exp \left[(x, y)-\|y\|^{2} / 2\right]$ is the Radon-Nikodym derivative of the normal distribution translated by $y$ with respect to the normal distribution on $H$. Thus if $f$ is a bounded continuous tame function, $\left(T_{y} f\right)(x)=f(x-y) D(x, y)$ is an integrable tame function of $x$ for each $y$ and $\left\|T_{y} f\right\|_{p}=\|f\|_{p}$. Since the bounded continuous tame functions are dense in $L^{p}(H)$ for $1 \leqq p<\infty$, the $T_{y}$ extend to isometries of $L^{p}(H)$. The following theorem is basic to all of the main results of this paper.

THEOREM 3. The map $y \rightarrow T_{y}$ from $H$ to the bounded linear operators on $L^{p}(H)$ is a strongly continuous representation of the additive group of $H$ as a group of isometries on $L^{p}(H)$.

Proof. The map $y \rightarrow T_{y}$ is a homomorphism of the additive group of $H$ onto a group of isometries on $L^{p}(H)$.

Since the bounded tame functions which are boundedly and continuously differentiable are dense in $L^{p}(H)$, an $\varepsilon / 3$-argument shows that it suffices to prove that $T_{y} f$ is a continuous function from $H$ to $L^{p}(H)$ for these tame functions in order to prove the continuity of $T_{y} g$ for all $g$ in $L^{p}(H)$. Thus we shall assume that $f$ is a bounded tame function with bounded and continuous first derivatives. Let $\left\{y_{n}\right\}$ be a sequence in $H$ converging to $y$ in the norm topology for $H$. Then

$$
\begin{aligned}
\left\|T_{y} f-T_{y_{n}} f\right\|_{p} \leqq\left(\int_{H} \mid\right. & \left.f(x-y)-\left.f\left(x-y_{n}\right)\right|^{p} D(x, y) d n(x)\right)^{1 / p} \\
& \quad+\left(\int_{H}\left|f\left(x-y_{n}\right)\right|^{p}\left|D_{p}(x, y)-D_{p}\left(x, y_{n}\right)\right|^{p} d n(x)\right)^{1 / p},
\end{aligned}
$$

where $D(x, y)=\exp \left[(x, y)-\|y\|^{2} / 2\right]$ and $D_{p}(x, y)=D(x, y)^{1 / p}$. By the mean value theorem, the first integral on the right side of (2.1) is dominated by a constant multiple of $\left\|y-y_{n}\right\|$; so the first term on the right of (2.1) tends to zero as $y_{n}$ tends to $y$ in $H$.

By the boundedness of $f$ and Hölder's inequality, the second term on the right of (2.1) is dominated by a constant multiple of $\left\|D_{p}(\cdot, y)-D_{p}\left(\cdot, y_{n}\right)\right\|_{k}$ where $k$ is an even integer greater than $p$. Now, expand $\left|D_{p}(x, y)-D_{p}\left(x, y_{n}\right)\right|^{k}$ by the binomial theorem and write $y_{n}=a_{n} y+b_{n} u_{n}$ where $a_{n}$ and $b_{n}$ are real numbers and $\left(y, u_{n}\right)=0$. Then we may use the fact that the orthogonal vectors $y$ and $u_{n}$ determine independent random variables to integrate the binomial series. The integral of this series is

$$
\sum_{j=1}^{k}(-1)^{j}\left(\begin{array}{l}
k \\
j
\end{array}\right) \exp \left[\left(j+a_{n}(k-j)\right)^{2} \frac{\|y\|^{2}}{2 p^{2}}+\left(b_{n}(k-j)\right)^{2} \frac{\left\|u_{n}\right\|^{2}}{2 p^{2}}-k \frac{\|y\|^{2}}{2 p}\right] .
$$

As $y_{n} \rightarrow y, a_{n} \rightarrow 1$ and $b_{n}^{2}\left\|u_{n}\right\|^{2} \rightarrow 0$. Thus the function above converges to

$$
\exp \left[\frac{1}{2}\left(p^{-2} k^{2}-p^{-1} k\right)\|y\|^{2}\right] \sum_{j=1}^{k}(-1)^{j}\left(\begin{array}{l}
k \\
j
\end{array}\right)=0
$$

and the second term on the right of (2.1) tends to zero as $y_{n}$ tends to $y$. 
We shall refer to the representation $y \rightarrow T_{y}$ of Theorem 3 as the regular representation of $H$ on $L^{p}(H)$.

Since, for $f \in L^{p}(H), T_{y} f$ is a continuous function from $H$ to the separable Banach space $L^{p}(H)$, we may define the integral operator determined by $v \in M(H)$ as the strong integral $\nu_{p}(f)=\int_{H} T_{y} f d \nu(y)$. There is the following

COROLlaRY 3.1. Let $1 \leqq p<\infty$ and $y \rightarrow T_{y}$ denote the regular representation of $H$ on $L^{p}(H)$. Then for $\nu$ in $M(H)$ the strong integral $\nu_{p}=\int_{H} T_{y} d \nu(y)$ is a bounded linear operator on $L^{p}(H)$ with norm at most $\|\nu\|$. The map $\nu \rightarrow \nu_{p}$ from $M(H)$ to the bounded linear operators on $L^{p}(H)$ is a uniformly continuous representation of the algebra $M(H)$ as an algebra of bounded operators on $L^{p}(H)$.

Proof. Since $T_{y} f$ is a bounded continuous function from $H$ to $L^{p}(H), T_{y} f$ is $\nu$-integrable for each $\nu$ in $M(H)$ and $\nu_{p}(f)=\int_{H} T_{y} f d \nu(y)$ satisfies

$$
\left\|\nu_{p}(f)\right\|_{p} \leqq \int_{H}\left\|T_{y} f\right\|_{p} d|\nu|(y) \leqq\|\nu\|\|f\|_{p} .
$$

The second statement follows directly from the fact that the map $y \rightarrow T_{y}$ is a strongly continuous homomorphism, and thus $\nu_{p} \circ \mu_{p}=(\nu * \mu)_{p}$ for $\nu$ and $\mu$ in $M(H)$, and the first statement of the corollary.

Denote by $\mathscr{F}$ the directed set of finite dimensional projections on $H$ and let $\nu$ be in $M(H)$. Then for $Q$ in $\mathscr{F}, \nu \circ Q^{-1}$ is in $M(H)$.

COROLlaRY 3.2. The integral operators $\left(\nu \circ Q^{-1}\right)_{p}$ converge strongly to $\nu_{p}$ as $Q$ tends strongly to the identity through $\mathscr{F}$.

Proof. Since $\left(\nu \circ Q^{-1}\right)_{p}(f)=\int_{H} T_{Q y} f d \nu(y)$, since $\left\|T_{y} f-T_{Q y} f\right\|_{p}$ tends to zero as $Q$ tends strongly to the identity through $\mathscr{F}$, and since the $T_{y}$ are isometries, the dominated convergence theorem implies that the $\left(\nu \circ Q_{n}^{-1}\right)_{p}$ converge strongly to $\nu_{p}$ for any sequence $\left\{Q_{n}\right\}$ of finite dimensional projections converging strongly to the identity. Since $L^{p}(H)$ is a metric space, this implies that $\left(\nu \circ Q^{-1}\right)_{p}$ converges strongly to $\nu_{p}$ as $Q$ tends strongly to the identity through $\mathscr{F}$.

We shall refer to the operators $\left(\nu \circ Q^{-1}\right)_{p}$, with $v$ in $M(H)$ and $Q$ in $\mathscr{F}$, as the tame integral operators.

In the case when $p=2$, we shall improve upon the estimate given in Corollary 3.1 for the norm of the operator $\nu_{2}$ on $L^{2}(H)$. Consider the Wiener transform $W(p)(y)=\int_{H} p\left(2^{1 / 2} x+i y\right) d n(x)$ which is defined initially on polynomials, $p(x)$, on $H$. Segal [9, pp. 119-122] has shown that the polynomials are dense in $L^{2}(H)$ and that the Wiener transform extends to a unitary operator on $L^{2}(H)$.

Corollary 3.3. Let $y \rightarrow T_{y}$ denote the regular representation of $H$ on $L^{2}(H)$, let $\nu$ be a complex Borel measure of bounded variation on $H$, and let $\nu_{2}=\int_{H} T_{y} d \nu(y)$ as above. If $W$ denotes the Wiener transform on $L^{2}(H)$, then

$$
W\left(\nu_{2} f\right)(\cdot)=W(f)(\cdot) \tilde{\nu}(1 / 2 \cdot)
$$


for each function $f$ in $L^{2}(H)$ where $\tilde{v}$ is the measurable function on $H$ corresponding to the Fourier transform, $\hat{v}(y)$, of $\nu$. Furthermore,

$$
\left\|\nu_{2}\right\|=\|\tilde{\nu}\|_{\infty}=\sup \{|\hat{\nu}(y)|: y \in H\} .
$$

Proof. Let $p$ be a polynomial on $H$. Then $W\left(T_{y} p\right)(z)=\exp [1 / 2 i(z, y)] W(p)(z)$ and $W\left(\nu_{2} p\right)(z)=W(p)(z) \hat{v}(1 / 2 z)$. Since $\nu_{2}$ and the Wiener transform are continuous on $L^{2}(H)$, it follows that $W\left(\nu_{2} f\right)(\cdot)=W(f)(\cdot) \tilde{\nu}(1 / 2 \cdot)$ for all $f$ in $L^{2}(H)$. Thus $\left\|v_{2}\right\|=\|\tilde{\nu}\|_{\infty}$. By Corollary 3.3 of [7], there is a one-one Hilbert-Schmidt operator $A$ on $H$ such that $\hat{\nu}(z)$ is uniformly continuous in the norm $\|A x\|$, and thus $\hat{v}(z)$ extends to a uniformly continuous function on the completion, $H_{A}$, of $H$ in this norm. But there is a Borel probability measure $N$ on $H_{A}$ and a map $F$ from $H^{*}$ to the measurable functions on $\left(H_{A}, S, N\right)$ which is a representative for the normal distribution, $n$, on $H$. Furthermore, this representative of the normal distribution is minimal. In this regard see Gross [8]. By Corollary 3 of [8], the measurable function $\tilde{v}(1 / 2 \cdot)$ determined by $\hat{v}(1 / 2 z)$ is equal almost everywhere to the extension of $\hat{\nu}(1 / 2 z)$ to $H_{A}$. Thus $\|\tilde{\nu}\|_{\infty}=\sup \{|\hat{\nu}(z)|: z \in H\}$.

3. Singular integral operators on $L^{2}(H)$. In this section we shall define the singular integral operators on $L^{p}(H)$ and study a class of singular integral operators on $L^{2}(H)$. In the following theorem we list the basic properties of the measures which we shall use to define the singular integral operators.

Theorem 4. If $\mu$ is in $M(H)$ and $t>0$, set $\mu_{t}=\mu \circ(t I)^{-1}$ where I denotes the identity on $H$. Then $\mu_{t}$ and the set function $\nu(\cdot)=\int_{a}^{b} \mu_{t}(\cdot) d t / t$ for $0<a<b<\infty$ are in $M(H)$. If $f(x)$ is a bounded continuous function from $H$ to a separable complex Banach space, then $\int_{H} f(x) d \nu(x)=\int_{a}^{b} \int_{H} f(t x) d \mu(x) d t / t$. The Fourier transform, $\hat{v}(y)$, of $\nu$ is given by $\hat{\nu}(y)=\int_{a}^{b} \hat{\mu}(t y) d t / t$, and the total variation of $\nu$ is dominated by $\|\mu\| \log (b / a)$.

Proof. That $\mu_{t}(\cdot)$ is in $M(H)$ is clear.

The class of complex valued Borel functions, $g$, on $H$ such that $g(t x)$ is Borel measurable on $(0, \infty) \times H$ is closed under pointwise limits and includes the continuous functions on $H$ and hence consists of all Borel functions. In particular if $E$ is a Borel set in $H, X_{E}(\cdot)$ is its characteristic function, and $\mu \in M(H)$, then $\mu_{t}(E)=\int_{H} X_{E}(t x) d \mu(x)$ is a measurable function of $t$. Thus $\nu(E)=\int_{a}^{b} \mu_{t}(E) d t / t$ is well defined, and by the bounded convergence theorem $\nu$ is countably additive.

For any Borel simple function $f$ on $H, \int_{H} f(x) d \nu(x)=\int_{a}^{b} \int_{H} f(t x) d \mu(x) d t / t$. For any bounded continuous function, $f$, on $H$ to a separable complex Banach space there is a uniformly bounded sequence of Borel simple functions, $f_{n}$, converging pointwise to $f$. By the bounded convergence theorem,

$$
\int_{H} f(x) d \nu(x)=\int_{a}^{b} \int_{H} f(t x) d \mu(x) d t / t .
$$

Since $\exp [i(y, x)]$ is a continuous function on $H$ for each $y$, the statement on the 
Fourier transforms follows from the equality of the integrals for continuous functions.

That $\|\nu\| \leqq\|\mu\| \log (b / a)$ follows from the equality

$$
\|\nu\|=\sup \left\{\left|\int_{H} f(x) d v(x)\right|:\|f\| \leqq 1\right\}
$$

where the functions $f$ are bounded, continuous, and complex valued.

Definition 3.1. Let $1 \leqq p<\infty$. For $0<\delta<\rho<\infty$ and $\mu \in M(H)$; set $Z^{\delta \rho}(\cdot)$ $=\int_{\delta}^{o} \mu_{t}(\cdot) d t / t$ and $Z_{p}^{\delta \rho} f=\int_{H} T_{y} f d Z^{\delta \rho}(y)$ where $y \rightarrow T_{y}$ denotes the regular representation of $H$ on $L^{p}(H)$. The $Z_{p}^{\delta \rho}$ are the approximate singular integral operators determined by $\mu$. If the strong limit, $Z_{p}$, of the $Z_{p}^{\delta \rho}$ exists as $\delta$ tends to zero and $\rho$ tends to infinity, then $Z_{p}$ is the singular integral operator determined by $\mu$.

Since $L^{p}(H)$ is a metric space, the strong limit, $Z_{p}$, of the $Z_{p}^{\delta \rho}$ exists if and only if for each pair of sequence $\left\{\delta_{n}\right\}$ and $\left\{\rho_{n}\right\}$ with $\delta_{n}$ tending to zero and $\rho_{n}$ tending to infinity, the sequence $Z_{p^{n}}^{\delta_{n} \rho_{n}}$ converges strongly to $Z_{p}$. We shall continue to use the notation $Z_{p}^{\delta \rho}$ but, henceforth, $\delta$ and $\rho$ will take their values in positive sequences $\left\{\delta_{n}\right\}$ and $\left\{\rho_{n}\right\}$ where $\delta_{n}$ tends to zero and $\rho_{n}$ tends to infinity. We shall obviate the fact that the strong limit, $Z_{p}$, is independent of the sequences $\left\{\delta_{n}\right\}$ and $\left\{\rho_{n}\right\}$ where it exists.

In this section we shall be concerned with the approximate singular integral operators $Z_{2}^{\delta \rho}$ on $L^{2}(H)$ and their strong limits. To state the main theorem of this section we need the following fact which is due to Gross [7]: If a map $F$ from $H^{*}$ to measurable functions on a probability space $(S, \Sigma, \mu)$ is a representative of the normal distribution on $H$ and if $B$ is a Hilbert-Schmidt operator on $H$, then the weak distribution with representative $F \circ B^{*}$ from $H^{*}$ to measurable functions on $(S, \Sigma, \mu)$ is a Borel probability measure on $H$. This measure is denoted by $n \circ B^{-1}$.

The main result of this section is

TheOREM 5. Let $B$ be a Hilbert-Schmidt operator on $H$, let $d \mu(y)=a(y) d n \circ B^{-1}(y)$ where $a \in L^{r}\left(H, n \circ B^{-1}\right)$ for some $r>1$, and suppose that $\int_{H} a(y) d n \circ B^{-1}(y)=0$. Then the approximate singular integral operators $Z_{2}^{\delta \rho}$ determined by $\mu$ converge strongly on $L^{2}(H)$ to a bounded linear operator $Z_{2}$ as $\delta$ tends to zero and $\rho$ tends to infinity. For $0<\delta<\rho<\infty$, there exists a finite constant $D_{r}$, which depends only on $r$, such that $\left\|Z_{2}^{\delta \rho}\right\| \leqq D_{r}\|a\|_{r}$ and $\left\|Z_{2}\right\|=\sup \{|\hat{Z}(x)|: x \in H\} \leqq D_{r}\|a\|_{r}$, where $\hat{Z}(x)$ is the pointwise limit of the Fourier transforms $\hat{Z}^{\delta \rho}(x)$ as $\delta$ tends to zero and $\rho$ tends to infinity. The Fourier transform, $\hat{Z}$, of the singular integral operator, $Z_{2}$, is in $C h_{0}^{B^{*}}(H)$, and $W\left(Z_{2} f\right)(\cdot)=W(f)(\cdot) \tilde{Z}(\cdot)$ where $W$ denotes the Wiener transform on $L^{2}(H)$ and $\tilde{Z}(\cdot)$ denotes the measurable function on $H$ determined by $\hat{Z}(x)$.

We need three lemmas in order to prove this theorem.

LEMMA 5.1. The approximate singular integral operators $Z_{2}^{\delta \rho}$ converge strongly to a bounded operator, $Z_{2}$, on $L^{2}(H)$ as $\delta$ tends to zero and $\rho$ tends to infinity if and only if the Fourier transforms $\tilde{Z}^{\delta \rho}(\cdot)$ of the measures $Z^{\delta \rho}(\cdot)$ converge boundedly and in 
measure to an element $\tilde{Z} \in L^{\infty}(H)$ as $\delta$ tends to zero and $\rho$ tends to infinity. In case of convergence, $W\left(Z_{2} f\right)(\cdot)=\tilde{Z}(\cdot) W(f)(\cdot)$ and $\left\|Z_{2}\right\|=\|\tilde{Z}\|_{\infty}$, where $W$ denotes the Wiener transform on $L^{2}(H)$.

Proof. By Corollary 3.3, $W\left(Z_{2}^{\delta \rho} f\right)(\cdot)=\tilde{Z}^{\delta \rho}\left(\frac{1}{2} \cdot\right) W(f)(\cdot)$. Thus if the Fourier transforms converge boundedly and in measure to $\tilde{Z} \in L^{\infty}(H)$, then the dominated convergence theorem implies that the approximate singular integral operators converge strongly to a bounded operator, $Z_{2}$, and $W\left(Z_{2} f\right)(\cdot)=\tilde{Z}(\cdot) W(f)(\cdot)$. Thus $\left\|Z_{2}\right\|=\|\tilde{Z}\|_{\infty}$.

Suppose that the $Z_{2}^{\delta \rho}$ converge strongly on $L^{2}(H)$. By setting $f=1$ and taking Wiener transforms, it is seen that the strong convergence implies that the net $\left\{\tilde{Z}^{\delta \rho}\right\}$ is Cauchy in $L^{2}(H)$. After the usual reduction to sequences, it follows that the $\tilde{Z}^{\delta \rho}$ converge to a function $\tilde{Z}$ in $L^{2}(H)$, and the $\tilde{Z}^{\delta \rho}$ converge to $\tilde{Z}$ in measure. By Corollary 3.3, $\left\|Z_{2}^{\delta \rho}\right\|=\left\|\tilde{Z}^{\delta \rho}\right\|_{\infty}$, and the uniform boundedness theorem implies that these norms are uniformly bounded; thus, $\tilde{Z} \in L^{\infty}(H)$.

When the function $\tilde{Z} \in L^{\infty}(H)$ exists, we refer to it as the measurable Fourier transform of the singular integral operator $Z_{2}$.

It follows from Lemma 5.1 and Corollary 3.3 that a necessary condition that the strong limit, $Z_{2}$, of the $Z_{2}^{\delta \rho}$ exists is that $\mu(H)=0$. For by Theorem $4, \hat{Z}^{\delta \rho}(y)$ $=\int_{\delta}^{o} \hat{\mu}(t y) d t / t$ and $\hat{Z}^{\delta \rho}(0)=\hat{\mu}(0) \log (\rho / \delta)$. By Corollary 3.3, $\left\|Z_{2}^{\delta \rho}\right\|=\left\|\tilde{Z}^{\delta \rho}\right\|_{\infty}$ $=\sup \left\{\left|\hat{Z}^{\delta \rho}(y)\right|: y \in H\right\}$; and the $\hat{Z}^{\delta \rho}(\cdot)$ are not uniformly bounded unless $\hat{\mu}(0)=\mu(H)=0$.

Lemma 5.2. Let $a(y)$ be a polynomial on $H$ which is based in the carrier of $B B^{*}$. Let $d \mu(y)=a(y) d n \circ B^{-1}(y)$, suppose that $\mu(H)=0$, and set $Z^{\delta \rho}(\cdot)=\int_{\delta}^{\rho} \mu_{t}(\cdot) d t / t$. Then

(i) The Fourier transform of $Z^{\delta \rho}$ is given by

$$
\hat{Z}^{\delta \rho}(y)=\int_{\delta}^{o} \exp \left[-t^{2} \frac{\left\|B^{*} y\right\|^{2}}{2}\right] W_{1 / 2}(a \circ B)\left(t B^{*} y\right) d t / t,
$$

where $W_{1 / 2}$ denotes the Wiener transform with variance parameter $1 / 2$.

(ii) The $\hat{Z}^{\delta \rho}(y)$ converge uniformly on $0<\varepsilon \leqq\left\|B^{*} y\right\| \leqq R<\infty$, as $\delta$ tends to zero and $\rho$ tends to infinity.

(iii) For $B^{*} y \neq 0$, the limit $\hat{Z}(y)=\lim \left\{\hat{Z}^{\delta \rho}(y): \delta \rightarrow 0, \rho \rightarrow \infty\right\}$ is a polynomial in $B^{*} y\left\|B^{*} y\right\|^{-1}$ and $\hat{Z}(y)=0$ and $B^{*} y=0$.

Proof. By Theorem 4, $\hat{Z}^{\delta \rho}(y)=\int_{\delta}^{o} \int_{H} \exp \left[i t\left(B^{*} y, x\right)\right] a \circ B(x) d n(x) d t / t . a \circ B(x)$ is a polynomial on $H$; suppose that $a \circ B(x)$ is based on $F$ and let $Q$ be the orthogonal projection from $H$ to $F$. Since $\left(Q^{\perp} B^{*} y, x\right)$ and $\left(Q B^{*} y, x\right)$ are independent, the inner integral is equal to $\exp \left[-t^{2}\left(\left\|Q^{\perp} B^{*} y\right\|^{2} / 2\right)\right] \int_{F} \exp \left[i t\left(Q B^{*} y, x\right)\right] b(x) d n(x)$ where $b(x)$ denotes the restriction of $a \circ B(x)$ to $F$ and $Q^{\perp}=I-Q$. A direct computation shows that the last integral is $\exp \left[-t^{2}\left(\left\|Q B^{*} y\right\|^{2} / 2\right)\right] W_{1 / 2}(a \circ B)\left(t B^{*} y\right)$. This proves (i).

Represent $\hat{Z}^{\delta \rho}(y)$ as in the conclusion of (i); then for $0<\delta<\rho<\infty$ and 
$0<\delta_{1}<\rho_{1}<\infty,\left|\hat{Z}^{\delta \rho}(y)-\hat{Z}^{\delta_{1} \rho_{1}}(y)\right| \leqq\left|\hat{Z}^{\delta \delta_{1}}(y)\right|+\left|\hat{Z}^{\rho \rho_{1}}(y)\right|$. Since $W_{1 / 2}(a \circ B)\left(t B^{*} y\right)$ is a polynomial which is divisible by $t$, for $y$ in the ellipse $\left\|B^{*} y\right\| \leqq R$ there is a polynomial $q(t)$ such that $t$ divides $q(t)$ and $\left|W_{1 / 2}(a \circ B)\left(t B^{*} y\right)\right| \leqq q(t)$ for all nonnegative $t$. If we replace $\left\|B^{*} y\right\|$ by $\varepsilon>0$ in the exponential factor of the integrand, the conclusion (ii) follows.

When $B^{*} y=0$ each $\hat{Z}^{\delta \rho}(y)=0$. Suppose that $B^{*} y \neq 0$; then by (i)

$$
\hat{Z}(y)=\lim _{\delta \rightarrow 0} \lim _{\rho \rightarrow \infty} \int_{\delta \| B^{*} y !}^{\rho: B^{*} y \|} \exp \left[-t^{2} / 2\right] W_{1 / 2}(a \circ B)\left(t B^{*} y\left\|B^{*} y\right\|^{-1}\right) d t / t .
$$

Thus the limit $\hat{Z}(y)=0$ when $B^{*} y=0$ and $\hat{Z}(y)$ is a polynomial in $B^{*} y\left\|B^{*} y\right\|^{-1}$ when $B^{*} y \neq 0$.

Lemma 5.3. Let $a(y)$ be a polynomial on $H$ which is based in the carrier of $B B^{*}$. Let $d \mu(y)=a(y) d n \circ B^{-1}(y)$, suppose that $\mu(H)=0$, and set $Z^{\delta \rho}(\cdot)=\int_{\delta}^{o} \mu_{t}(\cdot) d t / t$. Then

$$
\begin{aligned}
\lim \left\{\hat{Z}^{\delta \rho}(y): \delta \rightarrow 0\right. & , \rho \rightarrow \infty\}=\hat{Z}(y) \\
& =\int_{H}\left[i \pi / 2 \operatorname{sgn}\left(B^{*} y, x\right)-\log \left|\left(B^{*} y, x\right)\right|\right] a \circ B(x) d n(x)
\end{aligned}
$$

for $B^{*} y \neq 0$ and $\hat{Z}(y)=0$ when $B^{*} y=0$. Furthermore, $\left|\hat{Z}^{\delta \rho}(y)\right| \leqq D_{r}\|a\|_{r}$ for $r>1$ where $D_{r}$ is a finite constant which depends only on $r$.

Proof. By Theorem 4, $\hat{Z}^{\delta \rho}(y)=\int_{\delta}^{\rho} \int_{H} \exp \left[i t\left(B^{*} y, x\right)\right] a(B x) d n(x) d t / t$. Since $\hat{Z}^{\delta \rho}(y)=0$ when $B^{*} y=0$ for all $0<\delta<\rho<\infty$, we may assume that $B^{*} y \neq 0$. Since $\int_{H} a(B x) d n(x)=0$, the inner integral above is equal to

$$
\int_{H}\left(\exp \left[i t\left(B^{*} y, x\right)\right]-\exp \left[-t\left\|B^{*} y\right\|\right]\right) a(B x) d n(x) .
$$

Since the integrand of this last integral is a tame function of $x$ and jointly continuous in $x$ and $t, \hat{Z}^{\delta \rho}(y)=\int_{H} \int_{\delta \| B^{*} y^{*} \mid}^{\rho \rho !}(\exp [i t(\omega, x)]-\exp [-t]) d t / \operatorname{ta}(B x) d n(x)$, where $\omega=B^{*} y\left\|B^{*} y\right\|^{-1}$.

If we apply Cauchy's theorem to the region enclosed by the quadrilateral with vertices $\delta\left\|B^{*} y\right\|, \rho\left\|B^{*} y\right\|,-i \delta\left\|B^{*} y\right\|(\omega, x),-i \rho\left\|B^{*} y\right\|(\omega, x)$ it follows that the inner integral is dominated in absolute value by

$$
2\left(1+(\omega, x)^{2}\right)^{1 / 2} \int_{0}^{1}\left(t^{2}+(\omega, x)^{2}(t-1)^{2}\right)^{-1 / 2} d t .
$$

An elementary integration shows that this expression is dominated by $C|\log |(\omega, x)||$ where $C$ is a finite constant. It also follows from this application of Cauchy's theorem that the inner integral in the expression for $\hat{Z}^{\delta \rho}(y)$ converges pointwise in $y$ to $i \pi / 2 \operatorname{sgn}(\omega, x)-\log |(\omega, x)|$.

Since $|\log |(\omega, x)||$ is a tame function based on the line through $\omega$, Hölder's 
inequality implies that $\left|\hat{Z}^{\delta \rho}(y)\right| \leqq D_{r}\|a\|_{r}$ where $D_{r}=C\left(\int_{-\infty}^{\infty}|\log | t||^{s} d n(t)\right)^{1 / s}$ with $1 / r+1 / s=1$ and $C$ as above. Since $|\log |(\omega, x)|||a(B x)|$ is an integrable tame function, the dominated convergence theorem implies that

$$
\lim _{\delta \rightarrow 0} \lim _{\rho \rightarrow \infty} \hat{Z}^{\delta \rho}(y)=\hat{Z}(y)=\int_{H}[i \pi / 2 \operatorname{sgn}(\omega, x)-\log |(\omega, x)|] a(B x) d n(x)
$$

where $\omega=B^{*} y\left\|B^{*} y\right\|^{-1}$. Since $\int_{H} a(B x) d n(x)=0$, this last integral is equal to the desired one.

Proof of Theorem 5. The set $\mathscr{P}$ of polynomials which are based on the carrier of $B B^{*}$ is dense in $L^{2}\left(H, n \circ B^{-1}\right)$. Let $B=U A$ be the polar decomposition of $B$. If $f$ is orthogonal to this set of polynomials in $L^{2}\left(H, n \circ B^{-1}\right)$ then

$$
\int_{H} f(U x) p(U x) d n \circ A^{-1}(x)=0
$$

for all $p$ in $\mathscr{P}$. Since the carrier of $B B^{*}$ is the closure of the range of $B$ and $\mathrm{Cl}[R(B)]=U \mathrm{Cl}[R(A)], p(U x)$ is based in $\mathrm{Cl}[R(A)] . d n \circ A^{-1}$ is concentrated on $\mathrm{Cl}[R(A)]=K$. Let $C$ denote the restriction of $A$ to $K$ and let $K_{C}$ denote the completion of $K$ in the norm $\|C x\|$. Then $C$ is an isometry from $K_{C}$ to $K$. If $F$ from $K^{*}$ to measurable functions on $\left(K_{C}, \Sigma, N\right)$ is the Wiener space representative for the normal distribution on $K$, then $N \circ C^{-1}=n \circ C^{-1}$. The map $g(x) \rightarrow g(C x)$ from $L^{2}\left(K, n \circ C^{-1}\right)$ to $L^{2}\left(K_{C}, N\right)$ is an isometric isomorphism. The image of this map contains the polynomials which are based on the span of finitely many eigenvectors of $C$. Thus

$$
\int_{K_{C}} g(C x) q(C x) d N(x)=0
$$

for all polynomials, $q$, on $K$ implies that $g(C x)=0$ in $L^{2}\left(K_{C}, N\right)$. Because of the isometry, this implies that $g(x)=0$ in $L^{2}\left(K, n \circ C^{-1}\right)$. Thus $f=0$ in $L^{2}\left(H, n \circ B^{-1}\right)$ and the polynomials are dense.

It suffices to assume that $1<r \leqq 2$ since the general case will follow by Hölder's inequality. When $1<r \leqq 2$, the polynomials, $a(y)$, which are based in the carrier on $B B^{*}$ are dense in $L^{r}\left(H, n \circ B^{-1}\right)$. When $a(y)$ is such a polynomial with $\int_{H} a(y) d n \circ B^{-1}(y)=0$, Lemma 5.2(ii) and Theorem 2 imply that the Fourier transforms $\tilde{Z}^{\delta o}(\cdot)$ converge in measure to a function $\tilde{Z}(\cdot)$, and by Lemma 5.3, convergence takes place boundedly. By Lemma 5.1 , the operators $Z_{2}^{\delta \rho}$ converge strongly to an operator $Z_{2}$. By Lemma 5.3 and Corollary $3.4,\left\|Z_{2}^{\delta \rho}\right\| \leqq D_{r}\|a\|_{r}$ where $D_{r}$ depends only on $r$; thus $\left\|Z_{2}\right\| \leqq D_{r}\|a\|_{r}$.

For any approximate singular integral operator determined by a function $a(y) \in L^{r}\left(H, n \circ B^{-1}\right)$ with vanishing integral, $\left\|Z_{2}^{\delta \rho}\right\| \leqq\|a\|_{1} \log (\rho / \delta) \leqq\|a\|_{r} \log (\rho / \delta)$ by Theorem 4 and Corollary 3.1. Since the polynomials in $L^{r}\left(H, n \circ B^{-1}\right)$ are dense, $Z_{2}^{\delta \rho}$ is the uniform limit of a sequence of approximate singular integral operators 
determined by polynomials. Thus $\left\|Z_{2}^{\delta \rho}\right\| \leqq D_{r}\|a\|_{r}$, and the sequence of singular integral operators determined by these same polynomials converges uniformly to a bounded operator $Z_{2}$. An $\varepsilon / 3$-argument shows that the $Z_{2}^{\delta \rho}$ converge strongly to $Z_{2}$ as $\delta$ tends to zero and $\rho$ tends to infinity; thus $\left\|Z_{2}\right\| \leqq D_{r}\|a\|_{r}$.

Let $Z_{2}$ be a singular integral operator determined by a polynomial $a(y)$. Then the Fourier transform, $\hat{Z}(y)$, of $Z_{2}$ satisfies $|\hat{Z}(y)| \leqq D_{r}\|a\|_{r}$ by Lemma 5.3. $\hat{Z}(y)$ is a polynomial in $B^{*} y\left\|B^{*} y\right\|^{-1}$ for $B^{*} y \neq 0$ and $\hat{Z}(y)=0$ when $B^{*} y=0$. Thus if $Z_{2}$ is a singular integral operator determined by a function in $L^{r}\left(H, n \circ B^{-1}\right)$ with vanishing integral, the Fourier transform, $\hat{Z}$, of $Z_{2}$ is the uniform limit of a sequence of Fourier transforms of singular integral operators determined by polynomials; thus $\hat{Z} \in C h_{0}^{B^{*}}(H)$ by the definition of this space.

Let $B^{*}=U A$ be the polar decomposition of $B^{*}$. Let $V^{\prime}$ be the orthogonal projection onto the kernel of $A$ and let $V=I-V^{\prime}$. Let $C$ be a one-one HilbertSchmidt operator on $H$ such that $V C=A$. Then $\left\|B^{*} x\right\| \leqq\|C x\|$. The Fourier transforms $\hat{Z}^{\delta \rho}(y)$ of the approximate singular integral operators determined by $a(y) \in L^{r}\left(H, n \circ B^{-1}\right)$ are uniformly continuous in the norm $\|C x\|$ and extend to uniformly continuous functions on the completion, $H_{C}$, of $H$ in the norm $\|C x\|$. Choose the representative for the normal distribution which maps into the Borel measurable functions on the Wiener space $H_{C}$. Then by Corollary 3 of [8], $\hat{Z}^{\delta \rho}(y)$ on $H_{C}$ is equal almost everywhere to the measurable function $\tilde{Z}^{\delta \rho}(\cdot)$ determined by $\hat{Z}^{\delta \rho}(y)$ on $H$. If the approximate singular integral operator $Z_{2}^{\delta \rho}$ is determined by a polynomial, then it follows as in Lemma 5.2(ii) that the $\hat{Z}^{\delta \rho}(y)$ converge uniformly on the sets $0<\varepsilon \leqq\left\|B^{*} y\right\| \leqq R<\infty$ in $H_{C}$ to $\hat{Z}(y)$. Since $\hat{Z}^{\delta \rho}(y)=0=\hat{Z}(y)$ when $B^{*} y=0$, this implies that $\hat{Z}=\tilde{Z}$ almost everywhere on $H_{C}$ and $\|\tilde{Z}\|_{\infty}=\sup \left\{|\hat{Z}(y)|: y \in H_{C}\right\}$ $=\sup \{|\hat{Z}(y)|: y \in H\}$. The usual density argument can be used to prove this statement for the Fourier transform of a singular integral operator determined by any $a(y) \in L^{r}\left(H, n \circ B^{-1}\right)$ with vanishing integral.

REMARK. Theorem 5 holds under less restrictive hypotheses. It suffices to assume that $a(y) \in L \log ^{+} L\left(H, n \circ B^{-1}\right)$ and that the integral vanishes. To show that the Fourier transforms of the approximate singular integral operators converge boundedly and in measure, one works on the Wiener space $H_{C}$ which we used in the last part of the proof of Theorem 5.

The singular integral operators have the disadvantage that they generally map tame functions to nontame functions. We shall introduce a class of operators which are well related to the singular integral operators but which preserve tameness.

Definition 3.2. Let $\mu$ be in $M(H)$ and $Q$ be a finite dimensional orthogonal projection on $H$. Set $Z^{\delta \rho}(\cdot)=\int_{\delta}^{o} \mu_{t}(\cdot) d t / t$ and denote by $\left(Z \circ Q^{-1}\right)_{p}^{\delta \rho}$ the tame integral operator $\int_{H} T_{y} d\left(Z^{\delta \rho} \circ Q^{-1}\right)(y)$. The $\left(Z \circ Q^{-1}\right)_{p}^{\delta \rho}$ are the approximate tame singular integral operators determined by $\mu$. If the strong limit, $\left(Z \circ Q^{-1}\right)_{p}$, of the $\left(Z \circ Q^{-1}\right)_{p}^{\delta \rho}$ exists as $\delta$ tends to zero and $\rho$ tends to infinity, then $\left(Z \circ Q^{-1}\right)_{p}$ is the tame singular integral operator on $L^{p}(H)$ determined by $\mu$ and $Q$. 
THEOREM 6. Let $a(y)$ be in $L^{r}\left(H, n \circ B^{-1}\right)$ for some $r>1$, set $d \mu(y)=a(y) d n \circ B^{-1}(y)$, and suppose that $\int_{H} a(y) d n \circ B^{-1}(y)=0$. If $Q$ is a finite dimensional projection on $H$, the tame singular integral operator $\left(Z \circ Q^{-1}\right)_{2} f=\lim \left\{\left(Z \circ Q^{-1}\right)_{2}^{\delta \circ} f: \delta \rightarrow 0\right.$ and $\rho \rightarrow \infty\}$ exists as a bounded operator on $L^{2}(H)$. For $f$ in $L^{2}(H), W\left(\left(Z \circ Q^{-1}\right)_{2} f\right)(\cdot)$ $=W(f)(\cdot)(Z \circ Q) \sim(\cdot)$ where $(Z \circ Q)^{\sim}(\cdot)$ is the measurable function determined by the tame function $\hat{Z}(Q y)$ and $\hat{Z}(y)$ is the Fourier transform of the singular integral operator determined by $\mu$. As $Q$ tends strongly to the identity through the directed set of finite dimensional projections, the $\left(Z \circ Q^{-1}\right)_{2}$ converge strongly to $Z_{2}$, the singular integral operator determined by $\mu$.

Proof. The proof of the existence of the tame singular integral operator, $\left(Z \circ Q^{-1}\right)_{2}$, determined by $a(y)$ and $Q$ parallels the proof of Theorem 5 . Since for polynomials $p$ on $H, W\left(\left(Z \circ Q^{-1}\right)_{2}^{\delta \rho} p\right)(y)=W(p)(y) \hat{Z}^{\delta \rho}(1 / 2 Q y)$, and since $\hat{Z}^{\delta \rho}(Q y)$ $=0$ and when $B^{*} Q y=0$, it follows just as in the proof of Theorem 5 , that the Fourier transforms $\hat{Z}^{\delta \rho}(Q y)^{\sim}$ converge boundedly and in measure to $(Z \circ Q)^{\sim}(\cdot)$ as $\delta$ tends to zero and $\rho$ tends to infinity. Thus the tame singular integral operator determined by $a(y)$ and $Q$ exists, and $W\left(\left(Z \circ Q^{-1}\right)_{2} f\right)(\cdot)=W(f)(\cdot)(Z \circ Q)^{\sim}(\cdot)$ for all $f$ in $L^{2}(H)$. Also, $\left\|\left(Z \circ Q^{-1}\right)_{2}\right\|=\left\|(Z \circ Q)^{\sim}\right\|_{\infty} \leqq D_{r}\|a\|_{r}$ where $D_{r}$ is the constant in Theorem 5. Since $\hat{Z}(y) \in C h_{0}^{B^{*}}(H)$, the $(Z \circ Q)^{\sim}$ converge to $\tilde{Z}$ in measure by Theorem 1. By the dominated convergence theorem, this implies that the $\left(Z \circ Q^{-1}\right)_{2}$ converge strongly to $Z_{2}$ as $Q$ tends strongly to the identity through the directed set of finite dimensional projections on $H$.

EXAMPLES. Let $B$ be a one-one selfadjoint Hilbert-Schmidt operator on $H$ and let $\left\{e_{i}\right\}$ be an orthonormal basis of $H$ consisting of eigenvectors of $B$. Let $B e_{i}=b_{i} e_{i}$.

(1) Let $a(y)=b_{i}^{-1} b_{j}^{-1}\left(y, e_{i}\right)\left(y, e_{j}\right)-\delta_{i j}$. Then $a \in L^{r}\left(H, n \circ B^{-1}\right)$ for $1<r<\infty$ and $\int_{H} a(y) d n \circ B^{-1}(y)=0$. By Theorem 5, $a(y)$ determines a bounded singular integral operator on $L^{2}(H)$. By Lemma 5.2, this operator has Fourier transform $-\left(B y, e_{i}\right)\left(B y, e_{j}\right)\|B y\|^{-2}$.

(2) Let $a(y)=\left(2 \pi^{-1}\right)^{1 / 2} b_{j}^{-1}\left(y, e_{j}\right)$. Then $a(y) \in L^{r}\left(H, n \circ B^{-1}\right)$ for $1<r<\infty$ and the integral of $a(y)$ is zero. By Theorem 5, $a(y)$ determines a singular integral operator on $L^{2}(H)$. By Lemma 5.2 this operator has Fourier transform $i\left(B y, e_{j}\right)\|B y\|^{-1}$.

(3) Let $B$ be as above and $a(y)=\|y\|^{2}-\|B\|_{2}^{2}$. Then $a(y)$ has integral zero by Lemma 1.2 of [6] and $a(y) \in L^{r}\left(H, n \circ B^{-1}\right)$ for $1<r<\infty$. By using Theorem 6 and Lemma 5.2 it can be shown that the Fourier transform of the singular integral operator determined by $a(y)$ is $-\left\|B^{2} y\right\|^{2}\|B y\|^{-2}$.

4. Singular integral operators on $L^{p}(H)$. Let $1<p<\infty$ and $y \rightarrow T_{y}$ denote the regular representation of the additive group of $H$ as a group of isometries on $L^{p}(H)$. As in $\S 3$, set $Z^{\delta \rho}(E)=\int_{\delta}^{o} \mu(E / t) d t / t$ and $Z_{p}^{\delta \rho} f=\int_{H} T_{y} f d Z^{\delta \rho}(y)$ when $\mu$ is a Borel measure of bounded variation on $H$. We shall use the fact that each Borel measure of bounded variation on $H$ can be written as a sum of an odd and an even Borel measure of bounded variation. 
THEOREM 7. Let $\mu$ be an odd Borel measure of bounded variation on $H$. Then the approximate singular integral operators $Z_{p}^{\delta \rho}$ determined by $\mu$ converge strongly to a bounded operator $Z_{p}$ as $\delta$ tends to zero and $\rho$ tends to infinity and $\left\|Z_{p}\right\| \leqq G_{p}\|\mu\|$, where $G_{p}$ is a constant which depends only on $p$.

Proof. Let $f$ be a bounded continuous tame function. Since $\mu$ is odd,

$$
Z_{p}^{\delta \rho} f=1 / 2 \int_{\delta \leqq|t| \leqq \rho} \int_{H} T_{t y} f d \mu(y) d t / t .
$$

Since the integrand is jointly continuous in $t$ and $y$, we may interchange the order of integration and apply Minkowski's integral inequality. Thus

$$
\left\|Z_{p}^{\delta \rho} f\right\|_{p} \leqq 1 / 2 \int_{H}\left\|\int_{\delta \leqq|t| \leqq \rho} T_{t y} f d t / t\right\|_{p} d|\mu|(y) .
$$

Let $f(x)$ be based on $K_{1}$; then for each $y,\left(T_{t y} f\right)(x)$ is based on the span, $K$, of $K_{1}$ and $y$. Let $g$ denote the restriction of $f$ to $K$ and let $\omega=y\|y\|^{-1}$ when $y \neq 0$. Since $\int_{\delta \leqq|t| \leqq \rho}\left(T_{t y} f\right) d t / t=0$ when $y=0$, we may suppose that $y \neq 0$ and write

$$
\left\|\int_{\delta \leqq|t| \leqq \rho} T_{t y} f d t / t\right\|_{p}=\left(\int_{K}\left|\int_{\delta\|y\| \leqq|t| \leqq \rho \| y ! !} g(x-t \omega) D_{p}(x, t \omega) d t / t\right|^{p} d n(x)\right)^{1 / p} .
$$

Since the normal distribution on $K$ is orthogonally invariant, we may suppose that $K$ has an orthonormal basis $e_{1}, \ldots, e_{N}$ and that $e_{1}=\omega$. If we write the integral over $K$ as an iterated integral and write the first integral as

$$
(2 \pi)^{-1 / 2} \int_{-\infty}^{\infty}\left|\int_{\partial \||y| \leqq|t| \leqq \rho ! y ! !} g\left(x_{1}-t, x_{2}, \ldots, x_{N}\right) \exp -\frac{\left(x_{1}-t\right)^{2}}{2 p} d t / t\right|^{p} d x_{1},
$$

it follows from M. Riesz' theorem on the Hilbert transform that

$$
\left\|\int_{\delta \leqq|t| \leqq \rho} T_{t y} f d t / t\right\|_{p} \leqq G_{p}\|f\|_{p}
$$

where $G_{p}$ is a constant which depends only on $p$. Thus $\left\|Z_{p}^{\delta \rho} f\right\|_{p} \leqq G_{p}\|\mu\|\|f\|_{p}$. Since the bounded continuous tame functions are dense in $L^{p}(H)$, this last inequality holds for all $f$ in $L^{p}(H)$.

To prove strong convergence, first let $f$ be a bounded continuous tame function. Then for $0<\delta<\rho<\infty$ and $0<\varepsilon<R<\infty,\left\|Z_{p}^{\delta \rho} f-Z_{p}^{\varepsilon R} f\right\|_{p} \leqq\left\|Z_{p}^{\delta \varepsilon} f\right\|_{p}+\left\|Z_{p}^{\rho R} f\right\|_{p}$. As above,

$$
\left\|Z_{p}^{\delta \varepsilon} f\right\|_{p} \leqq 1 / 2 \int_{H}\left(\int_{K}\left|\int_{\delta\|y ! \leqq|t| \leqq \varepsilon ! y\| !} g(x-t \omega) D_{p}(x, t \omega) d t / t\right|^{p} d n(x)\right)^{1 / p} d|\mu|(y),
$$

and we may take $\omega=e_{1}$. Then if $\delta_{n}$ and $\varepsilon_{n}$ are two sequences of positive numbers which converge to zero,

$$
(2 \pi)^{-1 / 2} \int_{-\infty}^{\infty}\left|\int_{\delta_{n}|y| \leqq|t| \leqq \varepsilon_{n}\|y\|} g\left(x_{1}-t, x_{2}, \ldots, x_{N}\right) \exp \left[-\frac{\left(x_{1}-t\right)^{2}}{2 p}\right] d t / t\right|^{p} d x_{1}
$$


converges boundedly to zero for each $x_{2}, \ldots, x_{N}$ and each $y$ by M. Riesz' theorem on the Hilbert transform. Two applications of the dominated convergence theorem imply that $\left\|Z_{p}^{\delta_{n}} \varepsilon_{n} f\right\|_{p}$ tends to zero as $\delta_{n}$ and $\varepsilon_{n}$ tend to zero. Since $L^{p}(H)$ is a metric space, this implies that $\left\|Z_{p}^{\delta \varepsilon} f\right\|_{p}$ converges to zero as $\delta$ and $\varepsilon$ tend to zero. A similar argument shows that $\left\|Z_{p}^{\rho R} f\right\|_{p}$ converges to zero as $\rho$ and $R$ tend to infinity. Let $Z_{p} f=\lim \left\{Z_{p}^{\delta \rho} f: \delta \rightarrow 0\right.$ and $\left.\rho \rightarrow \infty\right\}$. Then if $f$ is a tame function as above and if $\|f\|_{p}=1,\left\|Z_{p} f\right\|_{p}=\lim \left\{\left\|Z_{p}^{\delta \rho} f\right\|_{p}: \delta \rightarrow 0\right.$ and $\left.\rho \rightarrow \infty\right\} \leqq G_{p}\|\mu\|$. Thus $Z_{p}$ is a bounded linear operator on $L^{p}(H)$. For any function $g$ in $L^{p}(H)$ and any $\varepsilon>0$ let $f$ be a bounded continuous tame function such that $\|g-f\|_{p}<\varepsilon$. Then $\left\|Z_{p} g-Z_{p}^{\delta \rho} g\right\|_{p} \leqq$ $\left\|Z_{p} g-Z_{p} f\right\|_{p}+\left\|Z_{p} f-Z_{p}^{\delta \rho} f\right\|_{p}+\left\|Z_{p}^{\delta \rho} f-Z_{p}^{\delta \rho} g\right\|_{p}$. The first and third terms on the right are dominated by $\varepsilon G_{p}\|\mu\|$ and the second term on the right tends to zero as $\delta$ tends to zero and $\rho$ tends to infinity. This completes the proof.

In the case in which the measure $\mu$ is even we shall assume that

$$
d \mu(y)=a(y) d n \circ B^{-1}(y)
$$

where $B$ is a Hilbert-Schmidt operator on $H$ and $a(y) \in L^{r}\left(H, n \circ B^{-1}\right)$ for some $r>1$. The following theorem gives the first result of this type which we shall prove.

THEOREM 8. Let $a(y)$ be an even bounded continuous tame function which is based in the carrier of $B B^{*}$ and let $A(y)=a(B y)$. Suppose that there is a finite dimensional subspace $F$ of $H$ such that $\int_{F} A\left(x_{1}, x_{2}\right) d n\left(x_{1}\right)=0$. Let $d \mu(y)=a(y) d n \circ B^{-1}(y)$ and $Z_{p}^{\delta \rho}$ be the approximate singular integral operators determined by $\mu$. Then the $Z_{p}^{\delta o}$ converge strongly on $L^{p}(H)$ to a bounded operator $Z_{p}$ and $\left\|Z_{p}\right\| \leqq K(r, p, n)\|a\|_{r}$ for $r>1$ where the constant $K(r, p, n)$ depends only on $r, p$, and the dimension, $n$, of $F$.

The proof of this theorem will be based on the following sequence of lemmas.

Lemma 8.1. Let $F$ be an n-dimensional Euclidean space and let $h(y)\|y\|^{-n}$ be a Calderon-Zygmund kernel on $F$. Suppose that $h(y)$ is a bounded function and let $f \in L^{r}(F, n)$ for some $r>1$ satisfy $\int_{F} f(x) d n(x)=0$. Set

$$
\left(H_{1}^{\delta \rho} f\right)(x)=\int_{\delta<\|y\|<\rho} f(x-y) D_{1}(x, y) h(y)\|y\|^{-n} d y .
$$

Then there exists a constant $D_{r}$ which depends only on $r$ and $n$ and not on $\delta$ and $\rho$, such that $\left\|H_{1}^{\delta \rho} f\right\|_{1} \leqq D_{r}\|f\|_{r}$. The limit $H_{1} f=\lim \left\{H_{1}^{\delta \rho} f: \delta \rightarrow 0\right.$ and $\left.\rho \rightarrow \infty\right\}$ exists in $L^{1}(F)$ and $\left\|H_{1} f\right\|_{1} \leqq D_{r}\|f\|_{r}$.

Proof. Let $G(x)$ be a continuously differentiable function with compact support in $F$ and vanishing Lebesgue integral over $F$. Set

$$
K^{\delta \rho}(G)(x)=\int_{\delta<\|y\|<\rho} G(x-y) h(y)\|y\|^{-n} d y .
$$

Then by Theorem 5 p. 103 of [1],

$$
\left\|K^{\delta \rho}(G)\right\|_{1} \leqq C \int_{F}|G(x)|\left(1+\log ^{+}\|x\|+\log ^{+}|G(x)|\right) d x+C
$$


where $C$ is a finite constant which depends only on the dimension of $F$. By Theorem 7, p. 108 of [1], $K^{\delta \rho}(G)$ converges in $L^{1}(F, d x)$ to $K(G)$ as $\delta$ tends to zero and $\rho$ tends to infinity and

$$
\|K(G)\|_{1} \leqq C \int_{F}|G(x)|\left(1+\log ^{+}\|x\|+\log ^{+}|G(x)|\right) d x+C .
$$

Let $f(x)$ be a continuously differentiable function with compact support in $F$ and suppose that $f$ has vanishing integral with respect to the normal distribution on $F$. Set $G(x)=f(x) \exp \left[-\|x\|^{2} / 2\right]$; then $G(x)$ satisfies the above conditions. Since

$$
\begin{aligned}
\left\|H_{1}^{\delta \rho} f\right\|_{1} & =(2 \pi)^{-n / 2}\left\|K^{\delta \rho}(G)\right\|_{1} \\
\left\|H_{1}^{\delta \rho} f\right\|_{1} & \leqq C(2 \pi)^{-n / 2}\left(\int_{F}|G(x)|\left(1+\log ^{+}\|x\|+\log ^{+}|G(x)|\right) d x+1\right) .
\end{aligned}
$$

Since $\log ^{+}|G(x)| \leqq \log ^{+}|f(x)|$, it follows from Hölder's inequality that $\left\|H_{1}^{\delta o} f\right\|_{1}$ $\leqq C_{1}\|f\|_{r}+C_{2}\|f\|_{r}^{r}+C_{3}$ where $C_{1}, C_{2}, C_{3}$ are finite constants which depend only on $r$ and the dimension of $F$. When $\|f\|_{r}=1,\left\|H_{1}^{\delta o} f\right\| \leqq D_{r}=C_{1}+C_{2}+C_{3}$. Since the continuously differentiable functions with compact support and vanishing integral are dense in the closed subspace of $L^{r}(F)$ consisting of functions with vanishing integrals, $\left\|H_{1}^{\delta \rho} f\right\|_{1} \leqq D_{r}\|f\|_{r}$ for all $f$ in $L^{r}(F), r>1$, which have vanishing integral.

If $f$ is continuously differentiable with compact support, then the $H_{1}^{\delta \rho} f$ converge in $L^{1}(F)$ to a function $H_{1}(f)$ since the $K^{\delta \rho}(G)$ converge in $L^{1}(F, d x)$. Thus $\left\|H_{1} f\right\|_{1}$ $\leqq D_{r}\|f\|_{r}$ and $H_{1}$ defines a bounded operator from the closed subspace of $L^{r}(F)$ which consists of functions with vanishing integral into $L^{1}(F)$. The usual density argument shows that $H_{1}^{\delta \rho} f$ converges to $H_{1} f$ for all $f$ in $L^{r}(F)$ with vanishing integral.

Let $F$ be an $n$-dimensional subspace of the real separable Hilbert space $H$. For $y$ in $F$ and a bounded continuous tame function $A(x)$, define $V_{y}(A)(x)=A(x-y) D(x, y)$ where $D(x, y)=\exp \left[(x, y)-\|y\|^{2} / 2\right]$. The $V_{y}$ extend to isometries of $L^{1}(H)$ and the map $y \rightarrow V_{y}$ is a strongly continuous representation of the additive group of $F$ as a group of isometries on $L^{1}(H)$. The proof of this fact parallels the proof of Theorem 3.

LEMMA 8.2. Let $A(x)$ be a bounded continuous tame function on $H$ and let $F$ be an $n$-dimensional subspace of $H$. Suppose that $\int_{F} A\left(x_{1}, x_{2}\right) d n\left(x_{1}\right)=0$. Let $h(y)\|y\|^{-n}$ be a Calderon-Zygmund kernel on $F$ with $h(y)$ a bounded function. Set $H_{1}^{\delta \rho}(A)(x)$ $=\int_{\delta<\|y\|<\rho}\left(V_{y} A\right)(x) h(y)\|y\|^{-n} d y$. Then for $r>1$ there exists a finite constant, $D_{r}$, which depends only on $r$ and the dimension of $F$ such that $\left\|H_{1}^{\delta o}(A)\right\|_{1} \leqq D_{r}\|A\|_{r}$. As $\delta$ tends to zero and $\rho$ tends to infinity, $H_{1}^{\delta \rho}(A)$ converges in $L^{1}(H)$ to a function $H_{1}(A)$ with $\left\|H_{1}(A)\right\|_{1} \leqq D_{r}\|A\|_{r}$.

Proof. Let $A(x)$ be based on the subspace $K_{1}$ of $H$ and let $K$ denote the span of $F$ and $K_{1}$. Then $H_{1}^{\delta o}(A)(x)$ is a tame function based on $K$. Let $B(x)$ denote the 
restriction of $A(x)$ to $K$. Then $\left\|H_{1}^{\delta \rho}(A)\right\|_{1}=\int_{K}\left|\int_{\delta<\|y\|<\rho}\left(V_{y} B\right)(x) h(y)\|y\|^{-n} d y\right| d n(x)$. Since $F$ is a closed subspace of $K$, write $K=F \oplus F^{\perp}$ and let $x=\left(x_{1}, x_{2}\right)$. Then

$$
\left\|H_{1}^{\delta o}(A)\right\|_{1}=\int_{F^{\perp}} \int_{F}\left|\int_{\delta<\|y\|<\rho} B\left(x_{1}-y, x_{2}\right) D\left(x_{1}, y\right) h(y)\|y\|^{-n} d y\right| d n\left(x_{1}\right) d n\left(x_{2}\right) .
$$

For each $x_{2}$, the inner integral is dominated by $D_{r}\left(\int_{F}\left|B\left(x_{1}, x_{2}\right)\right|^{r} d n\left(x_{1}\right)\right)^{1 / r}$ by Lemma 8.1. The convergence of the $H_{1}^{\delta o}(A)$ to $H_{1}(A)$ in $L^{1}(H)$ follows from the convergence in Lemma 8.1 and the dominated convergence theorem.

Now it is necessary to consider a class of Calderon-Zygmund operators on $L^{p}(H), 1<p<\infty$. Let $C$ be a one-one linear operator from an $n$-dimensional subspace $F$ of $H$ into $H$. Let $\left(S_{y} f\right)(x)=f(x-C y) D_{p}(x, C y)$ for each $y$ in $F$ and each bounded continuous tame function $f$ on $H$. Here,

$$
D_{p}(x, C y)=\exp \left[(x, C y) / p-\|C y\|^{2} / 2 p\right] .
$$

The $S_{y}$ extend to isometries on $L^{p}(H)$ and the map $y \rightarrow S_{y}$ from $F$ to bounded operators on $L^{p}(H)$ is a strongly continuous representation of the additive group of $F$ as a group of isometries on $L^{p}(H)$.

Let $e_{1}, \ldots, e_{n}$ be an orthonormal basis for $F$ and let

$$
K^{(j)}(y)=\pi^{-(n+1) / 2} \Gamma((n+1) / 2)\left(y, e_{j}\right)\|y\|^{-(n+1)}
$$

on $F$. Let $K(y)$ denote the generic $K^{(j)}(y)$ and let $K_{\varepsilon r}(y)=K(y)$ if $0<\varepsilon<\|y\|<r<\infty$ and $K_{\varepsilon r}(y)=0$ otherwise. Let $R_{p}^{\varepsilon r}(f)=\int_{F} S_{y} f K_{\varepsilon r}(y) d y$ for $f \in L^{p}(H)$; the $R_{p}^{\varepsilon r}(f)$ are bounded operators on $L^{p}(H)$.

LEMMA 8.3. Let $R_{p}^{\varepsilon r}$ be as above. There is a finite constant $G_{p}$ which depends only on $p$ such that $\left\|R_{p}^{\varepsilon r} f\right\|_{p} \leqq n G_{p}\|f\|_{p}$ for all $f \in L^{p}(H) ; n$ is the dimension of $F$. The $R_{p}^{\text {er }}$ converge strongly on $L^{p}(H)$ to a bounded operator $R_{p}$ and for each $f \in L^{p}(H)$, $\sum_{j=1}^{n} R_{p}^{(j)}\left[R_{p}^{(j)}(f)\right]=-f$.

Proof. Write the integral $R_{p}^{\varepsilon r} f$ in polar coordinates. Since $K_{\varepsilon r}(y)$ is an odd function,

$$
R_{p}^{\varepsilon r}(f)=1 / 2 \int_{\varepsilon \leqq|t| \leqq r} \int_{\Sigma}\left(S_{t \omega} f\right) K(\omega) d \sigma(\omega) d t / t .
$$

Since $S_{t \omega} f$ is jointly continuous in $t$ and $\omega$, we may reverse the order of integration. Let $f(x)$ be a bounded continuous tame function. Then the inner integral is

$$
\int_{\varepsilon \leqq|t| \leqq r} f(x-t C \omega) D_{p}(x, t C \omega) d t / t .
$$

Since $C \omega \neq 0$, set $s=t\|C \omega\|$. The rest of the proof of the uniform boundedness of the $R_{p}^{\varepsilon r}$ and the strong convergence of the $R_{p}^{\varepsilon r}$ parallels the proof of Theorem 7 . We shall not repeat it. The estimate for the norm follows from the fact that 
$\left\|R_{p}^{\varepsilon r}\right\| \leqq G_{p} \int_{\Sigma}|K(\omega)| d \sigma(\omega)$, where $d \sigma$ denotes Lebesgue measure on the sphere, and fact that $|K(\omega)| \leqq \pi^{-(n+1) / 2} \Gamma((n+1) / 2)$.

To prove that $\sum_{j=1}^{n} R_{p}^{(j)}\left[R_{p}^{(j)}(f)\right]=-f$, let $f$ be a bounded continuous tame function; then $R_{p}^{(j)}(f)$ is a tame function on $H$. Let $W_{p / 2}$ denote the Wiener transform on the space $L^{2}\left(H, n_{p / 2}\right)$. It follows just as in the proof of Corollary 3.4 that $W_{p / 2}\left(R_{p}^{\varepsilon r} f\right)(y)=W_{p / 2}(f)(y) \int_{F} \exp [i(y, C x) / p] K_{\varepsilon r}(x) d x$. Extend $C$ to an operator $M$, on $H$ by defining $M\left(y_{1}+y_{2}\right)=C y_{1}$ for $y=y_{1}+y_{2} \in F \oplus F^{\perp}=H$. Then $M$ is continuous and the above Fourier transform is $\hat{K}_{\varepsilon r}\left(p^{-1} M^{*} y\right)$. For this kernel, it is well known that the Fourier transforms $\hat{K}_{\varepsilon r}(z)$ are uniformly bounded and converge to $i\left(z, e_{j}\right)\|z\|^{-1}$ for $z \neq 0$ and the limit is zero when $z=0$. Thus the $\hat{K}_{\varepsilon r}\left(p^{-1} M^{*} y\right)$ converge boundedly to the function $\hat{K}\left(M^{*} y\right)=i\left(M^{*} y, e_{j}\right)\left\|M^{*} y\right\|^{-1}$ for $M^{*} y \neq 0$ and $\hat{K}\left(M^{*} y\right)=0$ when $M^{*} y=0$. Since $C$ is not the zero operator, $M^{*} \neq 0$, and $\sum_{j=1}^{n} \hat{K}^{(j)}\left(M^{*} y\right)^{2}=-1$ when $M^{*} y \neq 0$ and this sum is equal to zero when $M^{*} y=0$. Since the kernel of $M^{*}$ is a proper subspace of $H, \sum_{j=1}^{n} \hat{K}^{(j)}\left(M^{*} y\right)^{2}$ is a tame function which is equal to -1 almost everywhere. Thus

$$
W_{p / 2}\left(\sum_{j=1}^{n} R_{p}^{(j)}\left[R_{p}^{(j)}(f)\right]\right)=-W_{p / 2}(f) \quad \text { and } \quad \sum_{j=1}^{n} R_{p}^{(j)}\left[R_{p}^{(j)}(f)\right]=-f
$$

for bounded continuous tame functions. Since the $R_{p}^{(j)}$ are continuous on $L^{p}(H)$, the desired identity holds for all $f$ in $L^{p}(H)$.

Let $B$ be the Hilbert-Schmidt operator in the statement of Theorem 8. Let $F$ be the $n$-dimensional subspace of $H$ such that $\int_{F} A\left(x_{1}, x_{2}\right) d n\left(x_{1}\right)=0$. Let $C$ denote the restriction of $B$ to $F$ and let $G$ be the image of $F$ under $B$. We may assume that $F$ is contained in the base of $A(y)$ and that $C$ is one-one on $F$. Let $P$ denote the orthogonal projection from $H$ onto $G$ and let $\mathscr{P}=\left\{P_{k}: P=P_{1}<P_{2}<P_{3}<\cdots\right\}$ be a sequence of finite dimensional projections on $H$ which converges strongly to the identity as $k$ tends to infinity. Let $Q \in \mathscr{P}, Q>P$, and consider the tame operator $\left(Z \circ Q^{-1}\right)_{p}^{\delta \rho}$ determined by the measure $\mu$ in Theorem 8 . Let $f$ be a bounded continuous tame function on $H$. Then

$$
\left(Z \circ Q^{-1}\right)_{p}^{\delta \rho} f=\int_{\delta}^{\rho} \int_{H}\left(T_{t Q B y} f\right) A(y) d n(y) d t / t .
$$

The function $\left(T_{t Q B y} f\right) A(y)$ is a tame function of $y$ and is based on a finite dimensional subspace, $J$, which we may assume contains $F$. Thus

$$
\left(Z \circ Q^{-1}\right)_{p}^{\delta \rho} f=\int_{\delta}^{\rho} \int_{J}\left(T_{t Q B y} f\right) A(y) d n(y) d t / t .
$$

LEMMA 8.4. Let $\left(Z \circ Q^{-1}\right)_{p}^{\delta \rho}$ be the approximate tame singular integral operator above and let $R_{p}$ be one of the operators in Lemma 8.3. Then if $f$ is a bounded continuous tame function,

$$
R_{p}\left[\left(Z \circ Q^{-1}\right)_{p}^{\delta \rho} f\right]=\left(H \circ Q^{-1}\right)_{p}^{\delta \rho} f=\int_{\delta}^{\rho} \int_{J}\left(T_{t Q B y} f\right) R_{1}(A)(y) d n(y) d t / t
$$


where $R_{1}(A)(y)$ is the limit in $L^{1}(H)$ of the $R_{1}^{\varepsilon r}(A)(y)=\int_{F}\left(V_{z} A\right)(y) K_{\varepsilon r}(z) d z$ as $\varepsilon$ tends to zero and $r$ tends to infinity.

Proof. Let $0<\varepsilon<r<\infty$. Then

$$
\begin{aligned}
R_{p}^{\varepsilon r}\left(\left(Z \circ Q^{-1}\right)_{p}^{\delta o} f\right) & =\left(Z \circ Q^{-1}\right)_{p}^{\delta \rho}\left(R_{p}^{\varepsilon r} f\right) \\
& =\int_{\delta}^{o} \int_{J}\left(R_{p}^{\varepsilon r} f\right)(x-t Q B y) D_{p}(x, t Q B y) A(y) d n(y) d t / t
\end{aligned}
$$

Since $Q B=C$ on $F$, it follows by direct computation that this integral is

$$
\int_{\delta}^{\infty} \int_{J}\left(T_{t Q B y} f\right) R_{1}^{\varepsilon / t r / t}(A)(y) d n(y) d t / t
$$

where $R_{1}^{\varepsilon / t r / t}(A)=\int_{F} V_{z}(A) K_{\varepsilon / t r / t}(z) d z$. Then

$$
\left\|\left(H \circ Q^{-1}\right)_{p}^{\delta \rho} f-R_{p}^{\varepsilon r}\left[\left(Z \circ Q^{-1}\right)_{p}^{\delta \rho} f\right]\right\|_{p} \leqq \delta^{-1}\|f\|_{p} \int_{\delta}^{\rho}\left\|R_{1}^{\varepsilon / t r / t}(A)-R_{1}(A)\right\|_{1} d t .
$$

By Lemma 8.2, the integrand is bounded independently of $\varepsilon, r$, and $t$, and for each $t$ in $\delta \leqq t \leqq \rho$, the integrand converges to zero as $\varepsilon$ tends to zero and $r$ tends to infinity. After the usual reduction to sequences, the dominated convergence theorem implies that the last integral converges to zero as $\varepsilon$ tends to zero and $r$ tends to infinity. This completes the proof.

Proof of Theorem 8. Let $f$ be a bounded continuous tame function. Let $B=U D_{1}$ be the polar decomposition of $B$. Let $V^{\prime}$ denote the projection onto the kernel of $D_{1}$ and $D$ be a one-one Hilbert-Schmidt operator such that $\left(I-V^{\prime}\right) D=D_{1}$. Let $H_{D}$ denote the completion of $H$ in the norm $\|D x\|$ and let the map $G$ from $H^{*}$ to measurable functions on the Wiener space $\left(H_{D}, \Sigma, N\right)$ be the representative of the normal distribution on $H$ as in [8]. $D$ is an isometry from $H_{D}$ to $H$ and the measure $N \circ D^{-1}$ on $H$ is $n \circ D^{-1} . R_{1}(A)(y)$ is a tame $L^{1}$-function on $H$; let $R_{1}(A)(y)$ $=f\left(\left(y, e_{1}\right), \ldots,\left(y, e_{n}\right)\right)$. The corresponding measurable function on $\left(H_{D}, \Sigma, N\right)$ is $f\left(G\left(e_{1}\right)(\bar{y}), \ldots, G\left(e_{n}\right)(\bar{y})\right)=R_{1}(A)^{\sim}(\bar{y})$. For $y$ in $H, f\left(G\left(e_{1}\right)\left(D^{-1} y\right), \ldots, G\left(e_{n}\right)\left(D^{-1} y\right)\right)$ $=R_{1}(A)^{\sim}\left(D^{-1} y\right)=b(y)$ is a Borel measurable function on $H$ which is in $L^{1}\left(H, n \circ D^{-1}\right)$ and

$$
\left(H \circ Q^{-1}\right)_{p}^{\delta \rho} f=\int_{\delta}^{\rho} \int_{H}\left(T_{t Q U V y} f\right) b(y) d n \circ D^{-1}(y) d t / t .
$$

Set $d \lambda(y)=b(y) d n \circ D^{-1}(y)$ and $\nu=\lambda \circ(U V)^{-1} . \nu$ is an odd measure and $\|\nu\|$ $=\left\|R_{1}(A)\right\|_{1} \leqq D_{r}\|A\|_{r}$ by Lemma 8.2. Thus $\left(H \circ Q^{-1}\right)_{p}^{\delta \rho} f=\int_{\delta}^{\rho} \int_{H}\left(T_{t Q y} f\right) d \nu(y) d t / t$. As $Q$ tends strongly to the identity through $\mathscr{P}, T_{t Q y} f$ converges in $L^{p}(H)$ to $T_{t y} f$ by the strong continuity of the group representation. The dominated convergence theorem implies that as $Q$ tends strongly to the identity through $\mathscr{P},\left(H \circ Q^{-1}\right)_{p}^{\delta \rho} f$ converges to $H_{p}^{\delta \rho} f=\int_{\delta}^{\rho} \int_{H} T_{t y} f d \nu(y) d t / t$. By Corollary 3.2, the tame operators $\left(Z \circ Q^{-1}\right)_{p}^{\delta \rho}$ converge strongly to the approximate singular integral operator $Z_{p}^{\delta \rho}$ 
as $Q$ tends strongly to the identity through $\mathscr{P}$. Since the operators $R_{p}^{(j)}$ are continuous, Lemma 8.3 implies that $-Z_{p}^{\delta \rho} f=\sum_{j=1}^{n} R_{p}^{(j)}\left[H_{p}^{(j) \delta \rho}(f)\right]$. Thus $\left\|Z_{p}^{\delta \rho} f\right\|_{p}$ $\leqq n G_{p} \sum_{j=1}^{n}\left\|H_{p}^{(j) \delta \rho}(f)\right\|_{p}$ by Lemma 8.3. By Theorem 7, $\left\|H_{p}^{(j) \delta o}(f)\right\|_{p} \leqq G_{p}\left\|\nu_{j}\right\|\|f\|_{p}$ where $v_{j}$ is the odd measure which determines $H_{p}^{(j) \delta \rho}$. By the estimate given above, $\left\|\nu_{j}\right\| \leqq D_{r}\|A\|_{r}=D_{r}\|a\|_{r} ;$ and the constant $D_{r}$ is independent of $j$. Then $\left\|Z_{p}^{\delta o} f\right\|_{p}$ $\leqq n^{2} G_{p}^{2} D_{r}\|a\|_{r}\|f\|_{p}$. Since the bounded continuous tame functions are dense in $L^{p}(H)$, this inequality holds for all $f$ in $L^{p}(H)$.

Since $\left\|Z_{p}^{\delta \rho} f-Z_{p}^{\varepsilon r} f\right\|_{p} \leqq n G_{p} \sum_{j=1}^{n}\left\|H_{p}^{(j) \delta \rho} f-H_{p}^{(j) \varepsilon r} f\right\|_{p}$, Theorem 7 implies that the $Z_{p}^{\delta \rho}$ converge strongly as $\delta$ tends to zero and $\rho$ tends to infinity. Set $K(r, p, n)$ $=n^{2} G_{p}^{2} D_{r} ;$ then if $\|f\|_{p}=1,\left\|Z_{p} f\right\|_{p}=\lim \left\{\left\|Z_{p}^{\delta \rho} f\right\|_{p}: \delta \rightarrow 0\right.$ and $\left.\rho \rightarrow \infty\right\} \leqq K(r, p, n)\|a\|_{r}$.

Denote by $E L_{0}^{r}(H ; B ; F)$ the closure in $L^{r}(H)$ of the bounded continuous even tame functions of the form $A(x)=a(B x)$ which have vanishing integral over the finite dimensional subspace $F$. Here, $a(x)$ is an element of $L^{r}\left(H, n \circ B^{-1}\right)$. Since $L^{r}\left(H, n \circ B^{-1}\right)$ is a Banach space, and since $\|A\|_{r}=\|a\|_{r}$ for these tame functions, each element in $E L_{0}^{r}(H ; B ; F)$ corresponds to one and only one element $a(y)$ in $L^{r}\left(H, n \circ B^{-1}\right)$. Each such function $a(y)$ is even and determines approximate singular integral operators which converge strongly on $L^{p}(H)$ to an operator $Z_{p}$ with norm at most $K(r, p, n)\|a\|_{r}$ when $r>1$. Denote the subspace of $L^{r}\left(H, n \circ B^{-1}\right)$ which corresponds to $E L_{0}^{r}(H ; B ; F)$ by $E L_{0}^{r}\left(H, n \circ B^{-1} ; F\right)$. We shall state the following corollary of Theorem 8 without further proof.

Corollary 8.1. Let $a(y) \in E L_{0}^{r}\left(H, n \circ B^{-1} ; F\right)$ and let $d \mu(y)=a(y) d n \circ B^{-1}(y)$. Then the approximate singular integral operators, $Z_{p}^{\delta \rho}$, determined by $\mu$ are uniformly bounded and converge strongly as $\delta$ tends to zero and $\rho$ tends to infinity to a bounded operator, $Z_{p}$, with $\left\|Z_{p}\right\| \leqq K(r, p, n)\|a\|_{r}$.

COROLlary 8.2. Let $a(y)$ be an even tame function in $L^{r}\left(H, n \circ B^{-1}\right)$ for some $r>1$ with $\int_{H} a(y) d n \circ B^{-1}(y)=0$. Let $d \mu(y)=a(y) d n \circ B^{-1}(y)$. Then the approximate singular operators, $Z_{p}^{\delta \rho}$, determined by $\mu$ are uniformly bounded and converge strongly to a bounded operator, $Z_{p}$, and $\left\|Z_{p}\right\| \leqq K\|a\|_{r}$, where $K$ is a constant which depends on $r, p$, and the dimension of the base space of $a(y)$.

Proof. Let $F$ denote the base space of $A(y)=a(B y)$. Since $A(y)$ is a tame function in $L^{r}(H)$ there is a sequence, $A_{n}$, of even bounded continuous tame functions which are based in $F$, which have vanishing integral with respect to the normal distribution, and which converge to $A(y)$ in $L^{r}(H)$. Since $n \circ B^{-1}$ is concentrated on the carrier of $B B^{*}$, we may suppose that the function $a(y)$ is based in the carrier of $B B^{*}$ so that $A(y)$ is based in the closure of the range of the absolute value of $B$. Then there is a bounded continuous tame function $a_{n}(y)$ which is based in the carrier of $B B^{*}$ such that $A_{n}(y)=a_{n}(B y)$ for each $n$. Thus $\left\|a-a_{n}\right\|_{r}=\left\|A-A_{n}\right\|_{r}$ tends to zero as $n$ tends to infinity.

Let $Z_{p}^{\delta \rho}$ be the approximate singular integral operators determined by the measure $d \mu(y)=a(y) d n \circ B^{-1}(y)$. By Corollary 3.1 and Theorem 4, $\left\|Z_{p}^{\delta \rho} f\right\|_{p}$ $\leqq\|a\|_{1} \log (\rho / \delta)\|f\|_{p} \leqq\|a\|_{r} \log (\rho / \delta)\|f\|_{p}$. Thus the sequence of approximate 
singular integral operators determined by the functions $a_{n}(y)$ converges uniformly to $Z_{p}^{\delta \rho}$ and this implies that $\left\|Z_{p}^{\delta \rho}\right\| \leqq K(r, p, \operatorname{dim}(F))\|a\|_{r}$. The sequence of singular integral operators determined by the $a_{n}(y)$ converges uniformly to a bounded operator $Z_{p}$ and an $\varepsilon / 3$-argument shows that the $Z_{p}^{\delta \rho}$ converge strongly to $Z_{p}$.

Let $a(y)$ be an even bounded continuous tame function which is based in the carrier of $B B^{*}$ and satisfies $\int_{H} a(y) d n \circ B^{-1}(y)=0$. Then if $A(y)=a(B y)$, $\int_{H} A(y) d n(y)=0$. Let $A(y)$ be based on $K$ and let $e_{1}, \ldots, e_{N}$ be an orthonormal basis for $K$. Extend this basis to an orthonormal basis, $\left\{e_{i}\right\}$, for all of $H$. Let $F_{1}$ denote the span of $e_{1}, \ldots, e_{n}$, let $F_{2}$ denote the span of $e_{n+1}, e_{n+2}, \ldots, e_{2 n}$, and let $F_{k}$ denote the span of $e_{(k-1) n+1}, \ldots, e_{k n}$. Then $H=\sum_{j=1}^{\infty} \oplus F_{j}$ and we write $y$ in $H$ as $y=y_{1}+y_{2}+y_{3}$ where $y_{1} \in \sum_{j=1}^{k-1} \oplus F_{j}, y_{2} \in F_{k}$, and $y_{3} \in \sum_{j=k+1}^{\infty} \oplus F_{j}$. Let $T_{k} A=\int_{F_{k}} A\left(y_{1}, y_{2}, y_{3}\right) d n\left(y_{2}\right)$. Then $A(y)=\sum_{j=1}^{k}\left(T_{j-1} \cdots T_{1} A-T_{j} \cdots T_{1} A\right)$ where $\operatorname{dim}(K) \leqq k n$. Let $A_{j}=T_{j-1} \cdots T_{1} A-T_{j} \cdots T_{1} A$. Then $T_{j} A_{j}=0$, and

$$
\|A\|_{r} \leqq \sum_{j=1}^{k}\left\|A_{j}\right\|_{r} \leqq 2 k\|A\|_{r} .
$$

Denote by $E N_{0}^{r}(H, B, e)$ the completion of the even bounded continuous tame functions of the form $A(y)=a(B y)$ which are based on the span of finitely many elements of the orthonormal basis $e=\left\{e_{i}\right\}$ in the norm $N_{r}(A)=\sum_{j=1}^{\infty}\left\|A_{j}\right\|_{r}$. To each element of $E N_{0}^{r}(H, B, e)$ there corresponds an even function in $L^{r}\left(H, n \circ B^{-1}\right)$ whose integral is zero. For let $A \in E N_{0}^{r}(H, B, e)$ and let $A_{n}(x)=a_{n}(B x)$ be the sequence of bounded continuous tame functions which converges to $A$. Then $\left\|a_{n}-a_{m}\right\|_{r}=\left\|A_{n}-A_{m}\right\|_{r} \leqq N_{r}\left(A_{n}-A_{m}\right)$. Since this last norm tends to zero as $n$ and $m$ tend to infinity, there is an element $a(y)$ of $L^{r}\left(H, n \circ B^{-1}\right)$ which is the limit of the $a_{n}(y)$. The correspondence is one-one.

Thus there is a subspace $E N_{0}^{r}\left(H, n \circ B^{-1}, e\right)$ of $L^{r}\left(H, n \circ B^{-1}\right)$ which consists of even functions with zero integral with respect to $n \circ B^{-1}$. Define the norm on this subspace by $N_{r}(a)=n_{r}(A)$ where $A$ is the function in $E N_{0}^{r}(H, B, e)$ which determines $a$ as above.

CoRollary 8.3. If $a(y) \in E N_{0}^{r}\left(H, n \circ B^{-1}, e\right)$ for some $r>1$ and some orthonormal basis $e$ for $H$, then the approximate singular integral operators, $Z_{p}^{\delta o}$, determined by $d \mu(y)=a(y) d n \circ B^{-1}(y)$ are uniformly bounded and converge strongly on $L^{p}(H)$ to a bounded operator, $Z_{p}$, as $\delta$ tends to zero and $\rho$ tends to infinity. $\left\|Z_{p}\right\|$ $\leqq K(r, p, n) N_{r}(a)$.

Proof. Let $a(y)$ be a bounded continuous tame function which is based in the carrier of $B B^{*}$ and let $A(y)=a(B y)$. Suppose that the integral of $A$ with respect to the normal distribution is zero. Write $A=\sum_{j} A_{j}$ as above and let $A_{j}(y)=a_{j}(B y)$. By Theorem 8, the measures $d \mu_{j}(y)=a_{j}(y) d n \circ B^{-1}(y)$ determine singular integral operators $Z_{p}^{(j)}$ with norm $\left\|Z_{p}^{(j)}\right\| \leqq K(r, p, n)\left\|a_{j}\right\|_{r}$, and the approximate singular integral operators determined by $a(y)$ have norms uniformly bounded by $K(r, p, n) N_{r}(a)$. For $a(y) \in N_{0}^{r}\left(H, n \circ B^{-1}, e\right)$, there is a sequence of bounded 
continuous tame functions, $a_{n}(y)$, which are based in the carrier of $B B^{*}$ such that the $a_{n}$ converge to $a$ in $N_{0}^{r}\left(H, n \circ B^{-1}, e\right)$. Just as in the proof of Corollary 8.2, it follows that the norms of the approximate singular integral operators determined by $a(y)$ are uniformly bounded by $K(r, p, n) N_{r}(a)$ and the approximate singular integral operators converge strongly.

REMARK. It can be seen from the proof of Lemma 8.1 that it suffices for the tame function $a(y)$, which determines the approximate singular integral operators $Z_{p}^{\delta \rho}$, to be an element of $L \log ^{+} L\left(H, n \circ B^{-1}\right)$ in order that the $Z_{p}^{\delta \rho}$ converge strongly.

5. Singular integrals and the Calderon-Zygmund theory. Let $E$ denote $N$ dimensional Euclidean space. The Calderon-Zygmund theorem states that the operator

$$
H(f)(x)=P \int_{E} f(x-y) h(y)\|y\|^{-N} d y
$$

is bounded on $L^{p}(E, d x)$ for $1<p<\infty$ when $h(y)$ is positively homogeneous of degree zero, $h(y)$ has surface integral zero over the sphere, and $h(y)$ is $r$-power integrable over the sphere for some $r>1$. The $P$ preceding the integral indicates that the integral is to be taken in the principal value sense.

Let $K$ be a $k$-dimensional subspace of $E$ and let $U$ be a linear isometry from $K$ into $E$. Let $h(y)\|y\|^{-k}$ be a Calderon-Zygmund kernel on $K$. Then

$$
L(f)(x)=P \int_{K} f(x-U y) h(y)\|y\|^{-k} d y
$$

is also a bounded operator on $L^{p}(E, d x)$ for $1<p<\infty$.

For $f$ in $L^{p}(E, n)$, let $(E f)(x)=f(x) \exp \left[-\|x\|^{2} / 2 p\right]$. Then the map $T \rightarrow E^{-1} T E$ from the bounded operators on $L^{p}(E, d x)$ to the bounded operators on $L^{p}(E, n)$ is an isomorphism and a homeomorphism of the strong operator topologies. Thus the operator $L(f)$, given above, corresponds to the operator

$$
L_{p}(f)(x)=P \int_{K}\left(S_{y} f\right)(x) h(y)\|y\|^{-k} d y
$$

on $L^{p}(E, n)$ where $\left(S_{y} f\right)(x)=f(x-U y) \exp \left[(x, U y) / p-\|U y\|^{2} / 2 p\right]$. The map $y \rightarrow S_{y}$ from $K$ to bounded operators on $L^{p}(E, n)$ is a strongly continuous representation of the additive group of $K$ as a group of isometries on $L^{p}(E)$. We shall continue to refer to the operators of the form $L_{p}$ as Calderon-Zygmund operators.

The following theorem contains the main results of this section.

THEOREM 9. Let $1<p<\infty$ and $1<r \leqq 2$. Let $B$ be a linear operator on $E$ with polar decomposition $B=U C$. Let $a(y) \in L^{r}\left(E, n \circ B^{-1}\right)$ and suppose that

$$
\int_{E} a(y) d n \circ B^{-1}(y)=0 .
$$


Let $Z_{p}$ be the singular integral operator determined by $a(y)$. Then there is a complex constant

$$
\alpha=\int_{E}-\log \|y\| a(y) d n \circ B^{-1}(y)
$$

and a Calderon-Zygmund kernel $S(a)(y)\|y\|^{-k}$ on $K$, the range of $C$, such that

$$
Z_{p}(f)=\alpha f+P \int_{K}\left(S_{y} f\right) S(a)(y)\|y\|^{-k} d y
$$

- where $\left(S_{y} f\right)(x)=f(x-U y) \exp \left[(x, U y) / p-\|U y\|^{2} / 2 p\right] . S(a)(y)$ is defined initially on polynomials $a(y)$ by

$$
\begin{aligned}
S(a)(y)= & (2 \pi)^{-k / 2}(\operatorname{det} C)^{-1}\left\|C^{-1} y\right\| y\left\|^{-1}\right\|^{-k} \\
& \times \int_{0}^{\infty} a\left(t U y\left\|C^{-1} y\right\|^{-1}\right) \exp \left[-t^{2} / 2\right] t^{k-1} d t
\end{aligned}
$$

where $a(U y)$ is restricted to $K$ and where $C$ is regarded as an operator on $K$. There is a finite constant $D(r, k, C)$ which depends only on $r, k$, and $C$ such that $\|S(a)\|_{r}$ $\leqq D(r, k, C)\|a\|_{r}$ where the r-norm of $S(a)$ is the norm in $L^{r}(\Sigma, \sigma)$ and the r-norm of $a(y)$ is the norm in $L^{r}\left(E, n \circ B^{-1}\right)$.

Proof. Since the map $T \rightarrow E^{-1} T E$, given above, from the bounded operators on $L^{p}(E, d x)$ to the bounded operators on $L^{p}(E, n)$ is an isomorphism and a homeomorphism of the strong operator topologies, it suffices to consider the corresponding operators on $L^{p}(E, d x)$. Thus we consider

$$
f * Z=\lim \left\{f * Z^{\delta \rho}: \delta \rightarrow 0 \text { and } p \rightarrow \infty\right\}
$$

where $Z^{\delta o}(\cdot)=\int_{\delta}^{o} \mu_{t}(\cdot) d t / t$ and $d \mu(y)=a(y) d n \circ B^{-1}(y)$.

Let $g(x)$ be a continuously differentiable function with compact support in $E$. Then $\int_{E} g(x-y) a(y) d n \circ B^{-1}(y)=\int_{K} g(x-U y) a(U y) d n \circ C^{-1}(y)$ for each $x$. Let $a(y)$ be a polynomial in $L^{r}\left(E, n \circ B^{-1}\right)$ with integral zero. Restrict $a(U y)$ to $K$, let $\varepsilon>0$ and write

$$
\begin{aligned}
g * Z^{\delta o}(x)= & \int_{\delta}^{o} \int_{\|y\| \leqq \varepsilon} g(x) a(U y / t) d n_{t} 2 \circ C^{-1}(y) d t / t \\
& +\int_{\delta}^{o} \int_{\|y\| \leqq \varepsilon}[g(x-U y)-g(x)] a(U y / t) d n_{t} 2 \circ C^{-1}(y) d t / t \\
& +\int_{\delta}^{o} \int_{\|y\|>\varepsilon} g(x-U y) a(U y / t) d n_{t} 2 \circ C^{-1}(y) d t / t
\end{aligned}
$$

Write the first term on the right of (5.1) in terms of the characteristic function of the $\varepsilon$-ball, use the fact that $\int_{K} f(x) d \mu_{t}(x)=\int_{K} f(t x) d \mu(x)$, and interchange the order of integration. After a simple substitution, it is seen that since $\int_{K} a(U y) d n \circ C^{-1}(y)$ $=0$, the first term on the right of $(5.1)$ is

$$
g(x) \int_{E}-\log \|y\| a(y) d n \circ B^{-1}(y)
$$


for all $0<\delta<\rho<\infty$. Similarly, the second term on the right of (5.1) is seen to be dominated in absolute value by a constant multiple of $\varepsilon$ and the constant depends only on the $L^{1}$-norm of $a(y)$ and the sup-norm of the gradient of $g$ for all $0<\delta<\rho<\infty$. For the third term on the right of (5.1), we interchange the order of integration, make the substitutions $u=t^{-1}$ and $s=u\left\|C^{-1} y\right\|$ and take the limit as $\delta$ tends to zero and $\rho$ tends to infinity. Set

$$
G_{\delta \rho}(y)=\int_{\delta}^{\rho}\left(2 \pi t^{2}\right)^{-k / 2} a(U y / t) \exp \left[-\left\|C^{-1} y\right\|^{2} / 2 t^{2}\right] d t / t .
$$

There is a constant $M$ such that $\left|G_{\delta \rho}(y)\right| \leqq M\|y\|^{-k}$ for all $\|y\|>\varepsilon$ and $0<\delta<\rho<\infty$. By the dominated convergence theorem, the third term on the right side of (5.1) converges to

$$
\int_{\|y\|>8} g(x-U y) S(a)(y)\|y\|^{-k} d y .
$$

Take the limit as $\varepsilon$ tends to zero.

If $f(x)$ is homogeneous of degree zero, $\int_{K} f(x) d n(x)=\int_{\Sigma} f(\omega) d \sigma(\omega)$ where $\Sigma$ is the unit sphere in $K$ and $\sigma$ denotes normalized Lebesgue measure on $\Sigma$. If we use this fact, the invertibility of $C$ on $K$, and the Minkowski integral inequality, it can be shown that $\|S(a)\|_{r} \leqq D(r, k, C)\|a\|_{r}$. Similarly, it can be shown that the integral of $S(a)(\omega)$ over the unit sphere with respect to Lebesgue measure is equal to a constant multiple of $\int_{K} a(U y) d n \circ C^{-1}(y)=0$.

Since the continuously differentiable functions with compact support are dense in $L^{p}(E, d x)$ and since the polynomials with integral zero are dense in the subspace of $L^{r}\left(E, n \circ B^{-1}\right)$ consisting of functions with zero integral, the proof is complete.

Corollary 9.1. Assume the notation of Theorem 9. If the linear operator $B$ is the identity on $E$ and if the function $a(y)$ is positively homogeneous of degree zero, $Z_{p}$ is a Calderon-Zygmund operator with kernel $\omega_{N}^{-1} a(y)\|y\|^{-N}$, where $\omega_{N}$ is the area of the unit sphere in E. Every Calderon-Zygmund operator arises in this way.

Proof. Since $a(y)$ is homogeneous of degree zero and has integral zero and since $\log \|y\|$ is a radial function, the constant $\alpha$ is zero. Assume first that $a(y)$ is a bounded continuous function in $y \neq 0$ which is homogeneous of degree zero and has zero integral over the sphere. By an argument similar to that given in the proof of Theorem 9, $S(a)(y)=\omega_{N}^{-1} a(y)$.

Thus there is an intimate relationship between the singular integral operators introduced in $\S \S 3$ and 4 and the operators of Calderon and Zygmund.

6. Applications. Let $\Delta$ denote the Laplacian on $N$-dimensional Euclidean space. A. Calderon and Zygmund [1] have shown that there exists a Calderon-Zygmund operator, $H$, over $E$ which satisfies

$$
\frac{\partial^{2}}{\partial x_{i} \partial x_{j}} \Delta^{-1} f=N^{-1} \delta_{i j} f+H(f)
$$


Thus if $f$ is in $L^{2}(E, d x)$, the second derivatives of $\Delta^{-1} f$ are also $L^{2}$-functions. This result provides a regularity theorem for solutions of the Laplace equation on $E$. We shall prove an analogous result on Hilbert space. In constructing the analogous situation on Hilbert space, we shall use the fact that the partial derivative, $\partial / \partial x_{i}$, is the infinitesimal generator of the translation semigroup in the direction $e_{i}$ in $N$-dimensional Euclidean space.

Let $H$ be a real separable Hilbert space and let $e_{1}, e_{2}, e_{3}, \ldots$ be an orthonormal basis for $H$. Let $y \rightarrow T_{y}$ denote the regular representation of the additive group of $H$ acting on $L^{2}(H)$. Let $A_{j}^{(2)}$ denote the infinitesimal generator of the translation semigroup $T_{t e}$; i.e. $A_{j}^{(2)}=\left.(d / d t)\left(T_{t e}\right)\right|_{t=0}$. Let $A_{j}=-i 2 A_{j}^{(2)}$ where $i=(-1)^{1 / 2}$. Each $A_{j}$ is a closed densely defined linear operator on $L^{2}(H)$. If $p(y)$ is a polynomial on $H$ and $W$ denotes the Wiener transform on $L^{2}(H)$, then $W\left(A_{j} p\right)(y)=\left(y, e_{j}\right) W(p)(y)$.

Let $\left\{b_{i}\right\}$ be a square summable sequence of positive real numbers and consider the operators $L_{n}=\sum_{j=1}^{n} b_{j}^{2} A_{j}^{2}$. The $L_{n}$ are closed and densely defined on $L^{2}(H)$ and for polynomials $p(x)$ on $H, W\left(L_{n} p\right)(y)=\left\|B P_{n} y\right\|^{2} W(p)(y)$ where $P_{n}$ is the orthogonal projection onto the span of $e_{1}, \ldots, e_{n}$. By an argument similar to that in Lemma 1.2 of [6], the tame functions $\left\|B P_{n} y\right\|^{\sim 2}$ converge in $L^{2}(H)$ to the measurable function $\|B(\cdot)\|^{\sim 2}$ on $H$ as $n$ tends to infinity. Thus the operators $L_{n}$ converge to a closed densely defined linear operator $L=\sum_{j=1}^{\infty} b_{j}^{2} A_{j}^{2}$ which satisfies $W(L f)(\cdot)=\|B(\cdot)\|^{2} W(f)(\cdot)$ for $f$ in its domain. Since $\|B x\|$ is nonzero for $x \neq 0$, the measurable function $\|B(\cdot)\| \sim$ is nonzero almost everywhere. Thus $L$ admits a closed densely defined inverse, $L^{-1}$, with $W\left(L^{-1} f\right)(\cdot)=\|B(\cdot)\|^{\sim-2} W(f)(\cdot)$ for $f$ in the domain of $L^{-1}$.

Let $B$ be the operator on $H$ defined by $B e_{i}=b_{i} e_{i}$. Set

$$
a(y)=b_{i}^{-1} b_{j}^{-1} \delta_{i j}-b_{i}^{-2} b_{j}^{-2}\left(y, e_{i}\right)\left(y, e_{j}\right) .
$$

Then $a(y)$ has integral zero with respect to $n \circ B^{-1}$ and $a(y) \in L^{2}\left(H, n \circ B^{-1}\right)$. By Theorem $5, a(y)$ determines a singular integral operator, $Z_{2}$, on $L^{2}(H)$. By the first example at the end of $\S 3, W\left(Z_{2} p\right)(y)=\left(y, e_{i}\right)\left(y, e_{j}\right)\|B y\|^{-2} W(p)(y)$ for all polynomials $p$ on $H$. Thus $W\left(Z_{2} p\right)(y)=W\left(A_{i} A_{j} L^{-1} p\right)(y)$ for all polynomials $p$ on $H$. Since the polynomials are dense in $L^{2}(H)$, and since the Wiener transform is an isometry, $Z_{2}=A_{i} A_{j} L^{-1}$ as operators on $L^{2}(H)$. Thus we have

THEOREM 10. $L=\sum_{j=1}^{\infty} b_{j}^{2} A_{j}^{2}$ is a closed densely defined operator on $L^{2}(H)$ which admits a closed densely defined inverse, $L^{-1}$. If $Z_{2}$ denotes the singular integral operator on $L^{2}(H)$ determined by the function $a(y)=b_{i}^{-1} b_{j}^{-1} \delta_{i j}-b_{i}^{-2} b_{j}^{-2}\left(y, e_{i}\right)\left(y, e_{j}\right)$, then $Z_{2}=A_{i} A_{j} L^{-1}$ on $L^{2}(H)$.

We shall have more to say concerning regularity theorems of this type in a forthcoming paper [5].

7. REMARKS 1. The question as to whether the results of this paper can be extended to nonseparable Hilbert space leads to some basic considerations. If 
$H$ is a nonseparable Hilbert space, $L^{p}(H)$ is nonseparable. Thus if $y \rightarrow T_{y}$ denotes the regular representation of the additive group of $H$ acting on $L^{p}(H)$ and if $f \in L^{p}(H)$, the function $T_{y} f$ from $H$ to $L^{p}(H)$ may fail to be essentially separably valued with respect to a Borel measure $\mu$ on $H$ [see 3, p. 147], hence, $T_{y} f$ may fail to be $\mu$-measurable. If a Borel measure $\mu$ of bounded variation is concentrated on a separable subspace of $H$, then $T_{y} f$ is continuous and $\mu$-essentially separably valued and hence $\mu$-measurable. The question as to whether every Borel measure of bounded variation on a Hilbert space is concentrated on a separable subspace has been studied. In this regard see [7, p. 61] and the reference given there. Gross [7, p. 22] has proved that the Fourier transform of a Borel measure of bounded variation on a Hilbert space, $H$, is also the Fourier transform of a Borel measure of bounded variation which is concentrated on a separable subspace of $H$.

The measure $n \circ B^{-1}$ which we used in Theorems 5,6 , and 8 are only defined on separable Hilbert spaces [7, Definition 4, p. 23].

If $B$ is a Hilbert-Schmidt operator on $H$, let $H_{1}$ denote the smallest closed subspace of $H$ which contains the closures of the ranges of $B$ and $B^{*} . H_{1}$ is separable. Restrict $B$ to act in $H_{1}$. Let $n$ denote the normal distribution on $H_{1}$ and $n \circ B^{-1}$ be the usual measure on $H_{1}$. Let $f(x)$ be a bounded continuous tame function on $H$ and set $\left(V_{y} f\right)(x)=f(x-y) D_{p}(x, y)$ for $y$ in $H_{1}$. Here

$$
D_{p}(x, y)=\exp \left[(x, y) / p-\|y\|^{2} / 2 p\right] .
$$

The $V_{y}$ extend to isometries on $L^{p}(H)$ and the map $y \rightarrow V_{y}$ is a strongly continuous representation of the additive group of $H_{1}$ as a group of isometries on $L^{p}(H)$. If $f \in L^{p}(H), V_{y} f$ is Borel measurable on $H_{1}$. Let $a(y) \in L^{r}\left(H_{1}, n \circ B^{-1}\right)$ for some $r \geqq 1$ and set $d \mu(y)=a(y) d n \circ B^{-1}(y)$ and $Z^{\delta \rho}(\cdot)=\int_{\delta}^{o} \mu_{t}(\cdot) d t / t$. In this setting the analogues of Theorems 5, 6, 7, and 8 hold. The proofs are the same in all essential respects.

Theorem 7 can be generalized further. Let $\mu$ be a complex Borel measure of bounded variation on the Hilbert space $H$. Let $y \rightarrow T_{y}$ denote the regular representation of the additive group of $H$ acting on $L^{p}(H) ; 1 \leqq p<\infty$. Let $1 / p+1 / q=1$, $f \in L^{p}(H)$, and $g \in L^{q}(H)$. Set $\left\langle T_{y} f, g\right\rangle=E\left(\left(T_{y} f\right) \bar{g}\right)$ where $E$ denotes the expectation functional of the normal distribution on $H$. Define $\mu_{p}$ as the weak integral; i.e. $\left\langle\mu_{p} f, g\right\rangle=\int_{H}\left\langle T_{y} f, g\right\rangle d \mu(y)$. The analogues of Corollaries 3.1 and 3.3 follow. To prove the norm equality in Corollary 3.3 , one uses the fact that the Fourier transform of $\mu$ is based on a separable subspace of $H$. The analogue of Theorem 7 holds in this setting and the proof is essentially the same as that given in $\$ 4$. In place of Minkowski's integral inequality, one uses the fact that

$$
\int_{\varepsilon \leqq i t \mid \leqq r}\left\langle T_{t \omega} f, g\right\rangle d t / t=\left\langle\int_{\varepsilon \leqq|t| \leqq r} T_{t \omega} f d t / t, g\right\rangle
$$

and Hölder's inequality.

2. The results of this paper hold for the spaces $L^{p}\left(H, n_{c}\right)$ when $H$ is a separable 
Hilbert space and $n_{c}$ denotes the normal distribution with variance parameter $c>0$. If $f$ is a bounded continuous tame function on $H$,

$$
\left(T_{y} f\right)(x)=f(x-y) \exp \left[(x, y) / p c-\|y\|^{2} / 2 p c\right] .
$$

The $T_{y}$ extend to isometries on $L^{p}\left(H, n_{c}\right)$ and the map $y \rightarrow T_{y}$ is a strongly continuous representation of the additive group of $H$ as a group of isometries on $L^{p}\left(H, n_{c}\right)$. Now the definitions and results are as above.

3. The results above hold for the real Banach spaces $L^{p}(H)$ when the measures $\mu$ which determine the singular integral operators are assumed to be real valued. In the case when we consider the $L^{2}$-theory of $\S 3$, we must use the facts that a measure, $\mu$, is real valued if and only if its Fourier transform is Hermitian $\left(\hat{\mu}(-y)^{-}=\hat{\mu}(y)\right)$ and that the real space $L^{2}(H)$ is isomorphic to the closure of the Hermitian polynomials $\left(p(-y)^{-}=p(y)\right)$ in the complex space $L^{2}(H)$.

\section{REFERENCES}

1. A. P. Calderon and A. Zygmund, On the existence of certain singular integrals, Acta Math. 88 (1952), 85-139.

2. - Algebras of certain singular operators, Amer. J. Math. 78 (1956), 310-320.

3. N. Dunford and J. Schwartz, Linear operators, Volumes 1, 2, Interscience, New York, 1958, 1963.

4. M. J. Fisher, Singular integrals on Hilbert space, Ph.D. Thesis, Cornell Univ., Ithaca, N. Y., 1966.

5. - Sobolev chains, singular integrals, and operators of differential type over a Hilbert space (to appear).

6. L. Gross, Integration and nonlinear transformations in Hilbert space, Trans. Amer. Math. Soc. 94 (1960), 404-440.

7. - Harmonic analysis on Hilbert space, Mem. Amer. Math. Soc., No. 46, 1963.

8. - Abstract Wiener spaces, Proc. Fifth Berkeley Sympos. on Math. Stat. and Prob.

9. I. E. Segal, Tensor algebras over Hilbert spaces, Trans. Amer. Math. Soc. 81 (1956), 106-134.

10. - Distributions in Hilbert space and canonical systems of operators, Trans. Amer. Math. Soc. 88 (1958), 12-41.

THE JOHNS HOPKINS UNIVERSITY, BALTimore, MARYLAND 